\title{
Fault controlled Carboniferous A-type magmatism in the proto-Andean foreland (Sierras Pampeanas, Argentina): Geochemical constraints and petrogenesis
}

\author{
Juan A. Dahlquist ${ }^{a, b, *}$, Pablo H. Alasino ${ }^{b, c}$, G. Nelson Eby ${ }^{d}$, Carmen Galindo ${ }^{e}$, César Casquet ${ }^{e}$ \\ aCTERRA-CONICET-LNC, Rv. Vélez Sasfield 1611, Pab. Ceol, X5036CCR-Córdoba. Argentina \\ E UNLaR, Rv. Dr. Rene Favaloro, 5300-La Rioja, Argentina \\ 'CRIIAR-CONICET, Rnillaco Entre Ríos y Mendoza, (5301), Rnillaco, La Rioja, Argentina \\ - Deparment of Environmental, Earth, and A tmospheric Sciences, University of Massachusetts, Lowell, Massachusetts 01854, USA \\ e Departamento de Petrología y Ceonuinica, Universidad Complutense, 28040 Madrid, Spain
}

\section{A R T I C L E I N F O}

\section{Article history:}

Received 30 April 2009

Accepted 12 Novem ber 2009

Available online 26 November 2009

\section{Keywords:}

Early Carboniferous

A-type magmatism

Geochemistry

Biotite chemistry

$\mathrm{Sr}-\mathrm{Nd}$ isotopes

Shear zones

\begin{abstract}
A B S T R A C T
The intrusion of granitoids into the Eastern Sierras Pampeanas in the Early Carboniferous took place after a long period of mainly compressional deformation that included the Famatinian (Ordovician) and Achalian (Devonian) orogenies. These granitoids occur as small scattered plutons emplaced in a dominant extensional setting, within older metamorphic and igneous rocks, and many of them are arranged along a reactivated large shear zone. A set of 46 samples from different granitic rocks: Huaco granitic complex, San Blas pluton, and the La Chinchilla stock from the Sierra de Velasco, Zapata granitic complex from Sierra de Zapata, and the Los Árboles pluton from Sierra de Fiambalá, display high and restricted $\mathrm{SiO}_{2}$ contents between 692 and 76.4 wt.\%. On both $\mathrm{FeO} /(\mathrm{FeO}+\mathrm{MgO})$ vs. $\mathrm{SiO}_{2}$ and $\left[\left(\mathrm{Na}_{2} \mathrm{O}+\mathrm{K}_{2} \mathrm{O}\right)-\mathrm{CaO}\right]$ vs. $\mathrm{SiO}_{2}$ plots the samples plot in the ferroan and alkaline-calcic to calco-alkaline fields $\left(\mathrm{FeO} /(\mathrm{FeO}+\mathrm{MgO})=0.88-1 \mathrm{D} \% ;\left(\mathrm{Na}_{2} \mathrm{O}+\mathrm{K}_{2} \mathrm{O}\right)-\mathrm{CaO}\right)=$ 6.3-8.3\%), thus showing an A-type granitoid signature. The high concentrations for the High Field Strength Elements (HSFE), such as $\mathrm{Y}, \mathrm{Nb}, \mathrm{Ga}, \mathrm{Ta}, \mathrm{U}, \mathrm{Th}$, etc. and flat REE patterns showing significant negative Eu anomalies are also typical features of A-type granites. Our petrogenetic model supports progressive fractional crystallization with dominant fractionation of feldspar and a source mineral assemblage enriched in plagioclase. Biotites have distinctive compositions with high $\mathrm{FeO} / \mathrm{MgO}$ ratios (7.8-61.5), F (360$5610 \mathrm{ppm})$, and $\mathrm{Cl}$ (120-1050 ppm). The $\mathrm{FeO} / \mathrm{MgO}$ ratios together with the $\mathrm{F}$ and $\mathrm{Cl}$ content of igneous biotites seem to reflect the nature of their parental host magmas and may be useful in identifying A-type granitoids. The isotopic data ( $\mathrm{Rb}-\mathrm{Sr}$ and $\mathrm{Sm}-\mathrm{Nd}$ ) confirm that the A-type granites represent variable mixtures of asthenospheric mantle and continental crust and different mixtures lead to different subtypes of A-type granite (illustrating the lack of consensus about A-type magma origin). We conclude that prominent shear zones play an important role in providing suitable conduits for ascending asthenospheric material and heat influx in the crust, a hypothesis that is in accord with other recent work on A-type granites.
\end{abstract}

\section{Introduction}

The Early Carboniferous granitoids of the Sierras Pampeanas (La Rioja and Catamarca province, Argentina) occur as restricted and scattered outcrops. Most of the granitoids were emplaced along a prominent shear zone (Höckenreiner et al., 2003). The intrusion of these granitoids with an A-type signature (Dahlquist et al., 2006) followed several periods of intense magmatic activity: Middle Cambrian (Pampean Orogen), Early-Middle Ordovician (Farnatinian Orogen), and Middle-Upper Devonian (Achalian Orogen). A variety of studies during the past 10 years have improved our understanding of the petrogenesis and timing of the Pampean and Famatinian

* Corresponding author. cicterra CONICET-unC, Av. Vẻlez Sarsfield 1611, Pab. Geol.. X5016CGA-Córdoba. Argentina.

E-mail address: jdahlquist@efn.uncor.edu (J.A. Dahlquist). granitoids, but the late Palaeozoic granites (i.e., Devonian and Carboniferous) emplaced after the metamorphic peak of the Farnatinian Orogeny have received relatively little attention. In particular. they were only recently (Dahlquist et al., 2007) recognized as a genetic group representative of a specific magmatic event. The genesis of these granites is not well constrained, and they have been considered as products of a crustal reheating process during a final phase of the Famatinian orogen (e.g. Llambias et al., 1998; Grissom et al., 1998; Höckenreineret al., 2003; Miller and Söllner, 2005; Grosse et al., 2009) or part of a distinctive orogen called the Achalian (e.g. Sims et al., 1998; Stuart-Smith et al., 1999; Siegesmund et al., 2004; Dahlquist et al., 2006; López de Luchi et al., 2007).

In this paper we present major and trace element data for 46 whole-rock samples, biotite compositions, and $\mathrm{Rb}-\mathrm{S} r$ and $\mathrm{Sm}-\mathrm{Nd}$ isotope data for four Carboniferous large granitic units in the Eastern Sierras Pampeanas of La Rioja and Catamarca provinces. 


\section{Geological setting}

Using geochronological data and association with major orogenic eprsodes, four main granitoid groups have been recog:ized in the Eastern Sierras Pampeanas: Gi=Middle Camorian granites (Pampean), Gii = Farly-Midd:e Ordovician granites $\{$ Famatinian $\}$, Giii $=$ MiddleUpper Devonian granites, and Giv=Early Carboniferous granites (Sims et al., 1998; Rapela and Pankhurst, 1998; kapela et al., 1998; Pankhurst et al., 1998, 2000; Dahlçu!s: and Galindo, 2004; Büttner et al., 2005; Miller and Söllner 2005; Dahlquist et al., 2007, 2008; Rapela et al., 2008; Grosse et al., 2009).

The 'arge amount of geochronolog!cal ta:a for the l'ampern ant Famatinian granirestepored dising rhe ast renyears has improved our knowledge of the timing of these groups, but the Late Paleozoic granites einjaced after the metamor pic pea of the Famatinian Orogeny have received relatively little attention. Sims et al. (1998), Stuart-Smith et al. (1999), and Siegesmund et al. (2004) considered the voluminous Devonian intrusive rocks in the Sierras de Córdoba and the eastern area of the Sierras de San Luis to be associated with Devonian low-grade shear zones, together defoning the Achalian Orogeny $(368+25 \mathrm{Ma}$ U-Pb zircon, Dorais et al. (1997); $393 \pm 5$ Ma U-Pb SHRIMP zircon, StuartSmith et al. (1999); $382 \pm 5$ Ma Siegesmund et al. (2004); $369 \pm 9 \mathrm{Ma}$ $\mathrm{Rb} / \mathrm{Sr}$ whole-rock, Pinotti et al. (2006); and $379 \pm 4$ and $369 \pm 3 \mathrm{Ma}$ U-Pb SHRIMP zircon; Kapela et al. (2008); Fig. 1). According to this inter pletation the Devonian granites, such as the Achala (Sims et al., 1998) and Cerro Áspero batholiths in Sierra de Córdoba (e.g. Pinotti etal., 2002, 2006), and the Las Chacras (S1egesmiund et al., 2004) and Renca (Stuart-Smith et al., 1999) batholiths in Sierra de San Luis (Fig. 1), ale not post-orogenic intrusions of the Famalinian Orogeny as py.oposed by kapela and Pankhurst (1998), but belong to a distinct tectonomagmatic event. In NW Argenrina this event produced extensive NNW-SSE trending ductile shear zones, including the Tnogasta-Pituil-Antinaco (TIPA) shear zone (Hockencine: et al., 2003, and references theiein), that reached amphibolite grate. The Devonian compressiona', event has also been related either to collision of the Chilenia terrane with the proto-Pacific margin (Sims et al., 1998) or to a final collision between the Famatinian :nag:natic arc and the Pampean hinterland (Höckenreiner et al., 2003). To be consistent with previous associations between the names of the orngenies and those for the associated granitoids, Dahlquist et al (2006) referred to all the Devonian and Carboniferous g!a:ites as Achalian granites (Fig. 1). Kecent:y, these granites have been described by Grosse et al. (2009) as "part of the group of Middle Devonian-Lower Carboniferous granites of Sierras Pampeanas". However, field and geochemical data for the Early Carboniferous grantes are indicative of an extensional within-pinte setting and noncompressive tectonic regime, not syn-icinematic emplacement as has been invoked for the Devonian granites of Sierras de Córdoba or San Luis (Sims et al., 1998; Stuart-Smith et al, 1999; Sipgesthund et al., 2004 j. Da'zlquisis: et al. (2007) proposed that the Early Carboniferous A-type granites in the Eastern Sierras Pampeanas indicate a new distinctive tectonothermal event, i.e., the A-type event of the Eastern Sierras Pampeanas (ATEESM). This distinct intrusive event preceded the late Carboniferous exhumation of deep metamorphic levels and deposition of the continental sediments of the Paganzo Group. In most cases the Carboniferous gratrices were emplaced at shallow depth and are dominated by facies with K-feldspai inegaliysts. They are relatively small, isolated, sub-circular plutons scattered throughout the Eastern Sierras Pampeanas without any obvious sparial arrangements (although most were emplaced along the reactivated shear zone Fig. 1) or deformation.

\section{Field occurrence and petregraphic descriptions}

Our stukly was rarred out on four representar!ye granilir bodies of Farly Carbonifern'ts age whic h outropin rhe Eastern Sierras Pampeanas of Catamaica and LaRioja provinces (Figs. 1 and 2). These granitic bodies
(Los Árboles p'ułna, San Blas pluton, Zapata granir ir complex, Huaco granitic complex) (Figs. 1 and 2) occur along the large TIPA shear zone (Höckenreiner et al., 2003, and references therein). Höckenreiner et al. (2003) have suggested that the U-Pb ages for apaste in the granitic rocks (meta-granodiorite $342 \pm 1.8$; mylonite $328 \pm 3 \mathrm{Ma}$ ) of the Sierra de Coplarabana (near to the TIPA shear zone, Fig. 1) are consistent with the age of the Carboniferous granites and document a Carboniferous heating process that affected the whole crust.

Jetrograpinic investigations were conducted on 85 samples collected from these four granitic bodies and several smaller granitic borlies - the La Florida pluton and El Salto stock in Sierra de Fiambalá and the La Chinchilla stock in Sierra de Velasco (Figs. 1 and 2). Modal data are plotted in Fis. 3 and reveal a dominant monzogranire composition, transitional tog:'a:rodiolite and syenogranite (modal data are found in a s'spplemeala'. electronic data table).

\subsection{Los Arboles pluton}

The Los Árboles p'utun (LAP) (Los Ratones pluton of Grissom et al. $(1998)$ ) is an ellipsoidal intrusive body approximately $70 \mathrm{~km}^{2}$ in area and is located in the central parr of the Sierca de Fiambala. The host rocks are dominantly migmatices-g:teisses overprinted by a mylonitic deformation (Grissom et al., 1998; Höckenreiner et al, 2003). The contact of the LAP with the surrounding metamnephir rocks is sharp and intrusive. Metamorphic blocks of meter-scale are found in the intrusive.

Two main facies have been !ecogrized: (1) felsic porphyritic biotiteamphibole granodiorite to mnomgranite, found main'y in the westem region of the pluton, which has microcline phenocrysts $(3 \times 1.8$ to $1.5 \times$ $0.9 \mathrm{~cm}$ ! and is 'ypical.y or ange in colour and (2) leucocratic equigranular bin:!te monongranite, recognized in the central-northeastern regina whith is rnarse-grained $(\sim 1$ rmi, equigranciar and white in colour. Zircon, monazite, apatite, allanite and oxides are accessory minerals in both igneous facies, although in different propor tions. Biotite, containing unidentified radioactive minerals that are surrounded by 'arge, black, plenchrnic halos, are common in the leucocratic facies. Poorly aligned microcline phenocrysts define a poorly developed fabric (interpreted as mag:natic in origin) which, where measurable, has an orientation of $\sim 340-350^{\circ}$

A small pluton and a stock, La Florida and El Salto, respertively, have been recognized in the southern part of the Sierra de Fiambala. The Florida pluton is a leucocratic biotite monzogranile to syenogranite with a pnrphynitic texture imirracline phenocrysts are $3 \times 1.5 \mathrm{~cm}$ to $1 \times 0.8 \mathrm{rm}$. The El Salto stock is a leucocratic biotite monongranite with an equigranular medium-grained $(-0.6 \mathrm{~cm})$ texture and shows no sign of deformation. Mineral proportions reveal a dominant monzogranite composition, transitional to granodincite for the granitic rocks of the Sierra de Fiambalá (Fig. 3).

\subsection{The San Blas pluton}

The San Blas pluton (SBP) (Báez et al., 2002; Dahlquist et al., 2006) is a semi-ellipsoidal intrusive body of approximately $100 \mathrm{~km}^{2}$ located in the northwestern part of Sierra de Velasco (F!gs. 1 and 2). The host rocks are Carboniferous (343 Ma) porphyritic graniroids of the Asha pluton (Báez et al., 2004) and mylonitic rocks of the TIPA shear zone (Fockenteinel et al., 2003). The contact of the SBP with the surrounding Famatinian graaita!ts of the TIPA shear zone is sharp and marked hy strong topographic relief. Distinreively, the SBP houses several meter-sized mylonitic xenoliths (Alasino, 2002). Moreover, the SBP lacks the penetrative mylonitic deformation of the TIPA shear zone, which is widely found in the porphyritic graniroids of the Sierra de Velasco (regional distribution of mylonites from Aceñolaza et al. 2000; Höckenreiner et al., 2003; Fig. 1).

The dominant rock types in the SBP are porphyritic, biotite monzogranite to syenngranite (Fig. 3) with accessory muscovite. Their petrography is characterized by large microcline crystals $3-15 \mathrm{~cm}$ 


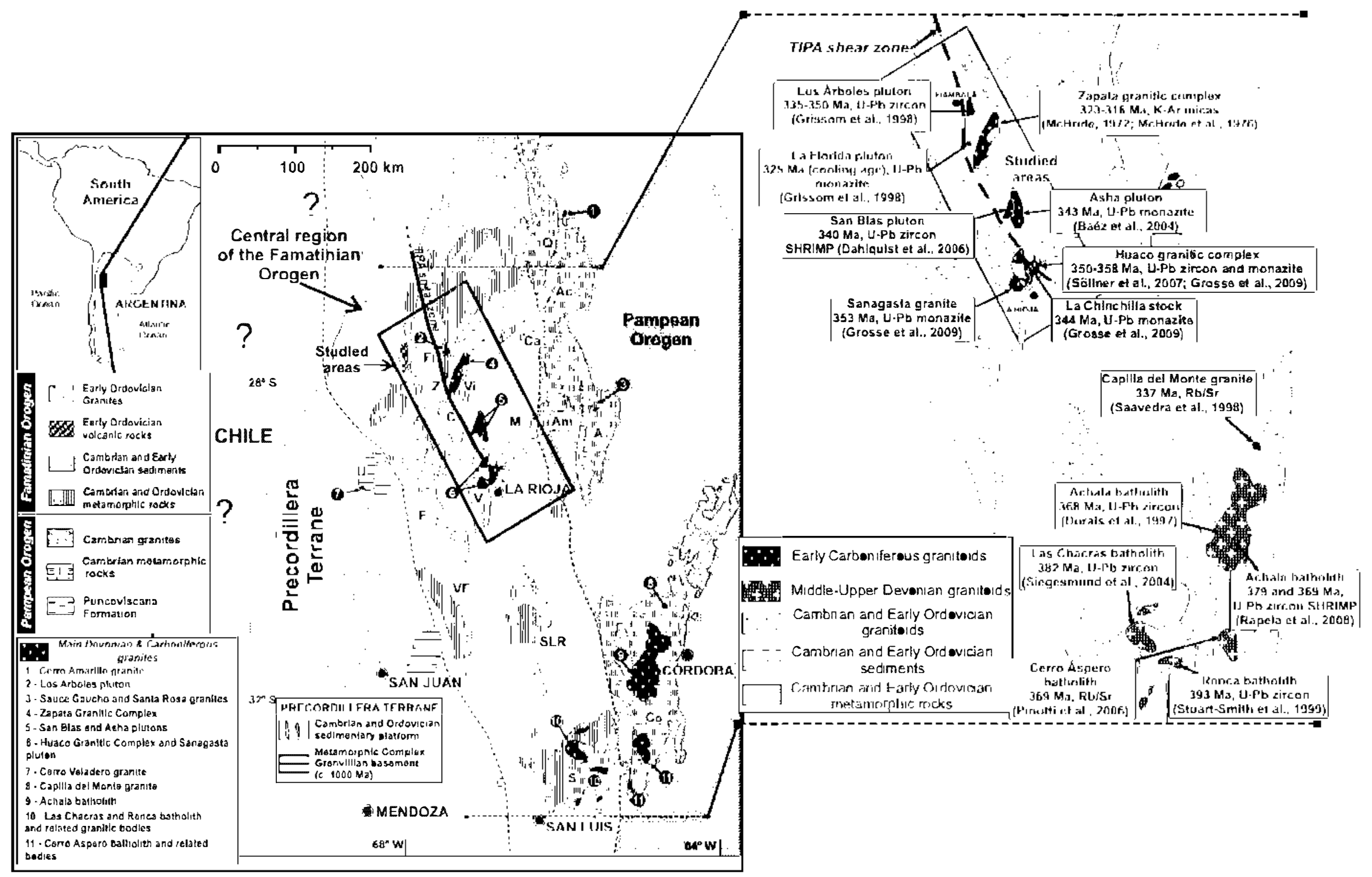

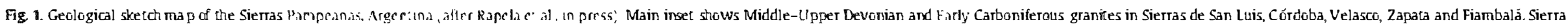

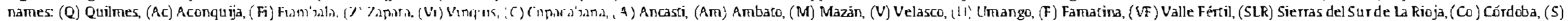
San Luis 


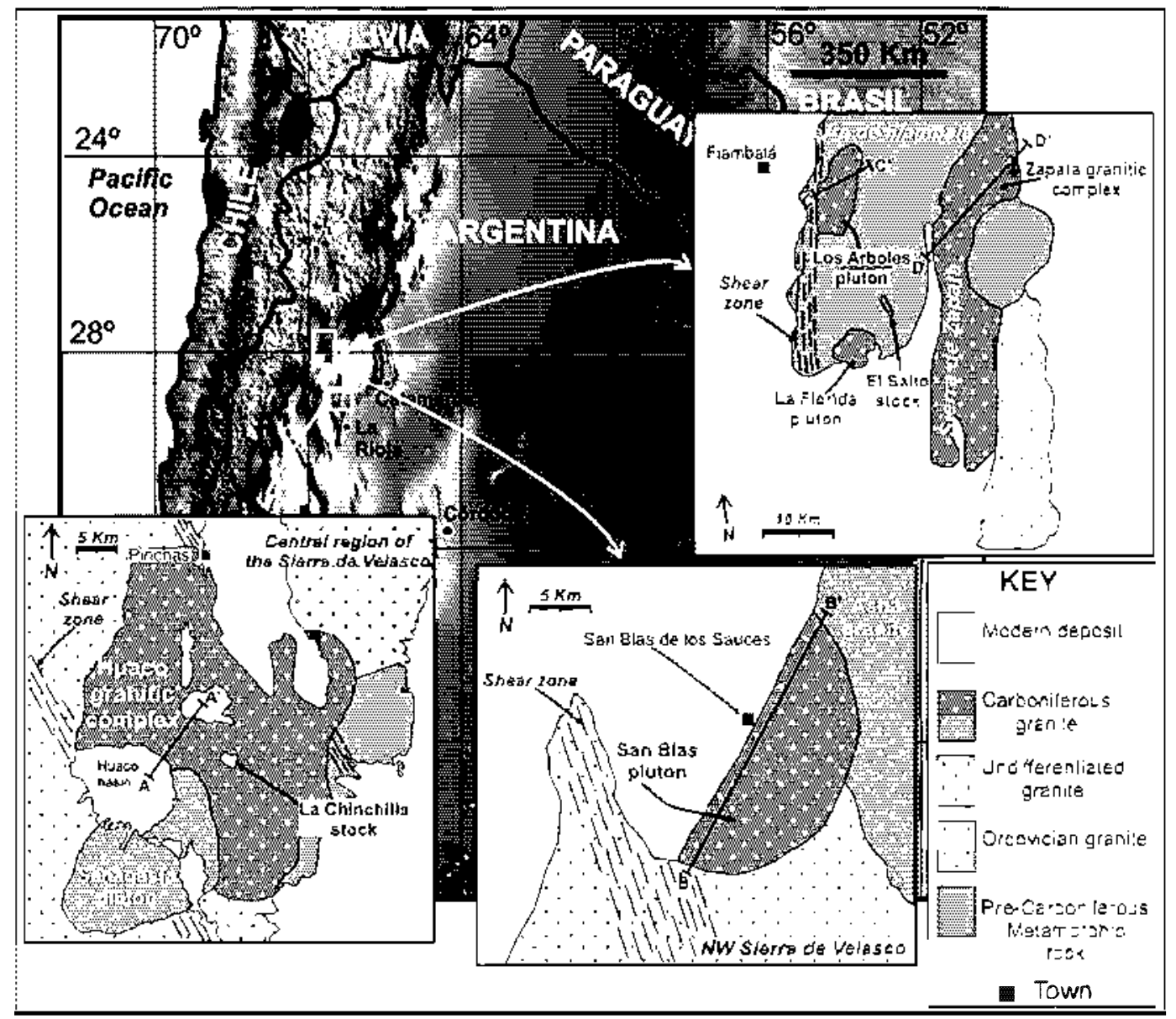

Fig. 2. IANDSA

region of the Sierra de Velasco is modified from Gro sse et al. (2009), the geologic map of the NW region of the Sierra de Velasco is modified from Dahlquist et al. (2006), and the geologic map of the Sierra de Fiambalá is modified from Grisso met al. (1998). The black lines (e.g. A-A', B-B', etc.) indicate the approximate locations of the samples ind uded in Table 1, excepting the small granitic bodies such as the La Forida and the El Salto pluton, and the La Chinchilla stock. Abbreviations in all the figures are: SF=Sierra de Fiambalá, $S Z=$ Sierra de Zapata, SV = Sierra de Velasco.

long), which constitute 6-35\% of the mode. The matrix is mediumgrained, equigranular, and mainly quartz, microcline, and biotite. Miariolitic textures have been observed. Recently, Alba (2008) recognized, according to a modal classification, three additional major facies: (1) feldspar porphyritic felsic granodiorite facies with slight alignment of microcline phen acrysts, localized in the rim of the pluton:

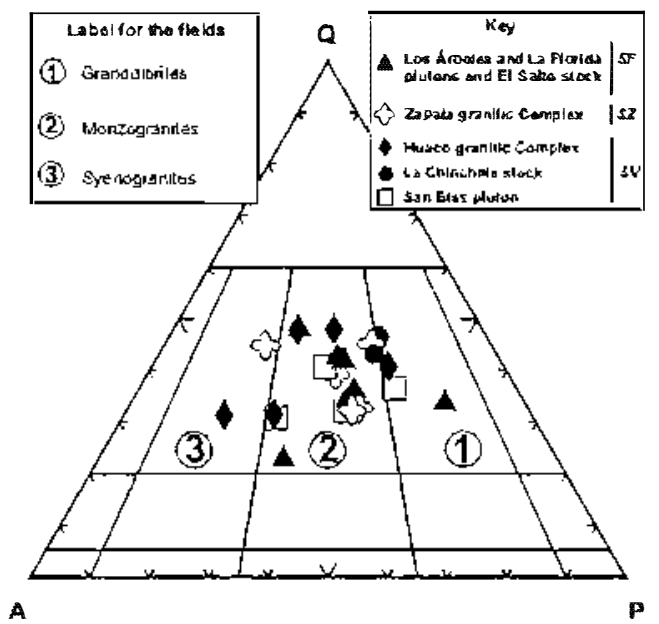

Fig. 3. Modal QAP diagram (Streckeisen, 1976) for the studied granitic rocks. $Q=$ quartz, $A=$ Alkali feldspar.
(2) the most common and dominant areal K-feldspar porphyritic monzogranite facies, found in the intermed iate region of the pluton; and (3) a slightly foliated $\mathrm{K}$-feld spar por phyritic syenogranite facies with a fine-grained matrix, lacalized in the central area. Felsic and mafic dykes cut the granites. Mafic microgranular enclaves have also been recognized (Báez et al., 2002), mainly in the northern region of the SBP (e.g. Navidad de los Cerros). Recently, a SHRIMP U-Pb zircon age of 340 Ma was reported far the Sam Blas pluton (Dahlquist et al., 2006).

\subsection{The Zapata granit ic conplex}

The Zapata granitic complex (ZGC) has an areal extent of approximately $470 \mathrm{~km}^{2}$ (Toselli et al., 1992). Along the studied cross-section (Figs. 1 and 2) metamorphic recles are very scarce, and they were only observed as small outcrops emergent in the modem sediments. The metamorphic rock is gneiss consisting of plagiclase, quartz, biotite and oxide minerals. The gneiss has an 'S' metamorphic fabric oriented $10 \%$ $59^{\circ} \mathrm{E}$. Numerous intrusive felsic veins were observed. The relationship between the metamorphic rocks and the ZGC is unlonown.

The dominant rock type in the ZGC is biotite monzogranite with zircon, mazite, apatite, oxides, \pm amphible and epid ote as accessory minerals. The texture is typically porphyritic with large microcline crystals ( 5.0 to $3.6 \mathrm{~cm}$ long), in a variable grain-sized matrix mainly consisting of quartz, micrecline, plagioclase, and biotite. Mineral proportions reveal a dominant monzogranite composition, transitional to syenogranite (Fig. 3). 
Representative major and trace element whole-rock data for the granitic complexes.

\begin{tabular}{|c|c|c|c|c|c|c|c|c|c|c|c|c|c|}
\hline \multirow[b]{3}{*}{ Facies } & \multicolumn{3}{|c|}{ Los Árboles pluton } & \multirow{3}{*}{$\begin{array}{l}\text { LFP } \\
P G\end{array}$} & \multirow[t]{3}{*}{ SVD } & \multirow{2}{*}{\multicolumn{5}{|c|}{ Zapata Granitic Co mplex }} & \multirow{2}{*}{\multicolumn{3}{|c|}{ Huaco Granitic Complex }} \\
\hline & \multicolumn{2}{|c|}{ Western Zone } & \multirow{2}{*}{$\frac{\text { Eastem Zone }}{E G}$} & & & & & & & & & & \\
\hline & $\mathrm{PG}$ & $P G$ & & & & Ap & $P G$ & FMPG & 56 & FMPG & $P G$ & FMPG & $m$ \\
\hline$\overline{\text { Locality }}$ & $\begin{array}{l}\overline{27^{\circ} 44^{\prime} 29^{\prime \prime}} \\
67^{\circ} 32^{\prime} 91^{\prime \prime}\end{array}$ & $\begin{array}{l}\overline{27^{\circ} 44^{\prime} 17^{\prime \prime}} \\
67^{\circ} 91^{\prime} 26^{\prime \prime}\end{array}$ & $\begin{array}{l}27^{\circ} 44^{\prime} 34^{\prime \prime} \\
67^{\circ} 30^{\prime} 04^{\prime \prime}\end{array}$ & $\begin{array}{l}\overline{27^{\circ} 58^{\prime} 50^{\prime \prime}} \\
67^{\circ} 32^{\prime} 58^{\prime \prime}\end{array}$ & $\begin{array}{l}\overline{27^{\circ} 44^{\prime} 92^{\prime \prime}} \\
67^{\circ} 92^{\prime} 96^{\prime \prime}\end{array}$ & $\begin{array}{l}27^{\circ} 53^{\prime \prime 56^{\prime \prime}} \\
67^{\circ} 22^{\prime} 22^{\prime \prime}\end{array}$ & $\begin{array}{l}27^{\circ} 59^{\prime} 99^{\prime \prime} \\
67^{\circ} 22^{\prime} 20^{\prime \prime}\end{array}$ & $\begin{array}{l}\overline{27} \cdot 59127 " \\
67^{\circ} 21^{\prime} 27^{\prime \prime}\end{array}$ & $\begin{array}{l}27^{\circ} 5917 " \\
67^{\circ} 21^{\prime} 15^{\prime \prime}\end{array}$ & $\begin{array}{l}\overline{27^{\circ} 27}\left(155^{\prime \prime}\right. \\
67^{\circ} 16^{\prime} 94^{\prime \prime}\end{array}$ & $\begin{array}{l}\overline{29^{\circ} 09^{\prime} 48^{\prime \prime}} \\
67^{\circ} 00^{\prime} 22^{\prime \prime}\end{array}$ & $\begin{array}{l}\overline{29^{\circ} 1 \cup 02^{\prime \prime}} \\
67000^{\prime \prime} 6^{\prime \prime}\end{array}$ & $\begin{array}{l}\overline{29^{\circ} 11^{\prime 23}} \\
67^{\circ} 01^{\prime \prime} 45^{\prime \prime}\end{array}$ \\
\hline$\overline{\text { Sample }}$ & FIA-C3 & FIA-22 & FIA-17 & FIA-24 & FIA-7 & $2 A P-27$ & $2 A P-29$ & $2 A P-32$ & 2AP-33 & $2 A P-36$ & HUA-01 & HUA-07 & HUA- 12 \\
\hline wer & & & & & & & & & & & & & \\
\hline $\mathrm{SiO}_{2}$ & 69.21 & 72.77 & 74.5 & 74.96 & 58.57 & 75.11 & 33.46 & 75.57 & 71.86 & 7334 & 71.05 & 7262 & 79.9 \\
\hline $\mathrm{TiO}_{2}$ & 0.40 & 0.37 & 0.07 & 0.14 & 1.61 & 0.06 & 0.13 & 0.14 & 0.25 & 0.12 & 0.23 & 0.19 & 0.19 \\
\hline $\mathrm{Al}_{2} \mathrm{O}_{3}$ & 1241 & 12.13 & 11.98 & 1209 & 1341 & 12.31 & 1232 & 11.66 & 13.1 & 1218 & 13.98 & 12.99 & 19.39 \\
\hline $\mathrm{Fe}_{2} \mathrm{O}_{3}$ & 3.69 & 393 & 1.45 & 1.86 & 7.56 & 1.91 & 1.99 & 201 & 241 & 2.04 & 2.9 & 209 & 227 \\
\hline $\mathrm{MnO}$ & 0.05 & 0.05 & 0.02 & 0.03 & 0.11 & 0.05 & $\bullet .04$ & 0.09 & 0.04 & 0.03 & .04 & 0.09 & 0.5 \\
\hline $\mathrm{MgO}$ & 0.46 & 0.91 & 0.03 & 0.11 & 275 & 0.05 & 0.1 & 009 & 0.19 & 0.07 & 0.27 & 0.22 & 0.15 \\
\hline $\mathrm{CaO}$ & 1.24 & 1.23 & 064 & 0.77 & 3.97 & 0.69 & 0.85 & 0.82 & 1.06 & - 88 & 1.04 & 0.6 & 084 \\
\hline $\mathrm{Na}_{2} \mathrm{O}$ & 3.00 & 2.86 & 3.29 & 299 & 3.08 & 3.27 & 318 & 2.91 & 3.06 & 3.29 & 3.12 & 2.81 & 3.18 \\
\hline$K \rightarrow$ & 5.07 & 5.09 & 4.92 & 482 & 3.22 & 5.49 & 5.18 & 509 & 5.7 & 498 & 5.51 & 5.7 & 5.19 \\
\hline $\mathrm{P}_{2} \mathrm{O}_{5}$ & 0.14 & 0.11 & 0.02 & 0.05 & 041 & 022 & 0.04 & 0.09 & 0.07 & 0.2 & 0.2 & 0.13 & 0.14 \\
\hline Lel & 289 & 1.89 & 1.66 & 1.76 & 431 & 1.92 & 1.66 & 1.66 & 1.79 & 1.84 & 1.54 & 1.59 & 1.69 \\
\hline Total & 98.5 & 100.14 & 98.58 & 99.52 & 99.00 & 100.29 & 98.89 & 99.95 & 9947 & 98.79 & 99.28 & 98.85 & 100.33 \\
\hline$(\mathrm{ppm})$ & & & & & & & & & & & & & \\
\hline $\mathrm{Be}$ & 4 & 6 & 6 & 8 & 6 & 7 & 8 & 7 & 7 & 7 & 12 & 7 & 8 \\
\hline sc & 5 & 5 & 2 & 5 & 14 & 5 & 3 & 4 & 5 & 4 & 4 & 3 & 4 \\
\hline$v$ & 18 & 14 & $<5$ & $<5$ & 196 & 8 & 6 & 6 & 8 & -5 & 9 & 11 & $<5$ \\
\hline$C_{T}$ & 170 & $x$ & 140 & 110 & 50 & 100 & 260 & 160 & 50 & 6 & 80 & 120 & 170 \\
\hline$C_{0}$ & 5 & 3 & $<1$ & $i$ & 18 & 2 & $i$ & l & 2 & $<1$ & 2 & 2 & 1 \\
\hline $\mathrm{Cu}$ & 140 & 10 & $<10$ & $<10$ & 20 & 30 & 10 & 10 & $<10$ & $<10$ & $<10$ & 20 & $x$ \\
\hline $2 n$ & 60 & 50 & $<30$ & $<30$ & 90 & 50 & 40 & 30 & 40 & 30 & 60 & 60 & 60 \\
\hline$C_{5}$ & 3.8 & 4.7 & 10.1 & 9.2 & 68 & 144 & 123 & 14 & 11.5 & 12 & 249 & 13.5 & 31.7 \\
\hline $\mathrm{Rb}$ & 235 & 300 & 650 & 467 & 292 & 429 & 511 & 470 & 372 & 556 & 408 & 420 & 450 \\
\hline ST & 91 & 81 & 9 & 18 & 219 & 20 & 32 & 27 & 74 & 25 & 79 & 49 & 60 \\
\hline $\mathrm{Ba}$ & 326 & 286 & 31 & 64 & 393 & 55 & 116 & 81 & 395 & 88 & 267 & 184 & 251 \\
\hline La & 93.4 & 87 & 53.2 & 57.6 & 62.7 & 111 & 59.6 & 89 & 53.4 & 99.8 & 41.5 & 41.9 & 51.6 \\
\hline $\mathrm{Ce}$ & 195 & 180 & 121 & 197 & 197 & 251 & 149 & 198 & 118 & 291 & 96.7 & 102 & 120 \\
\hline $\mathrm{PT}$ & 20 & 18.6 & 13.7 & 15.2 & 15.1 & 27.9 & 16.8 & 21.4 & 13.2 & 25.9 & 113 & 11.8 & 19.4 \\
\hline $\mathrm{Nd}$ & 67.1 & 61.7 & 51.5 & 55.7 & 59.9 & 92.3 & 64.5 & 71.5 & 463 & 89.1 & 41.6 & 436 & 49.1 \\
\hline$s m$ & 12.1 & 11.2 & 121 & 124 & 113 & 17.1 & 15.1 & 14.9 & 9.71 & 19.5 & 862 & 9.75 & 10.5 \\
\hline Eu & 1.13 & 108 & 0.18 & 0.32 & 1.7 & 0.82 & 0.5 & 0.42 & 1.1 & 0.48 & 1.05 & 0.66 & 1.19 \\
\hline Gd & 9.76 & 921 & $12 \mathrm{~A}$ & 12.2 & 10.1 & 13.6 & 13.9 & 11.3 & 8.65 & 16.2 & 7.21 & 8.17 & 8.05 \\
\hline$T b$ & 1.79 & 1.66 & 286 & 275 & 1.9 & 2.34 & 2.77 & 2.13 & 1.79 & 3.24 & 14 & 1.59 & 1.62 \\
\hline Dy & 9.7 & 9.5 & 19.1 & 17.9 & 11.2 & 12.5 & 17.0 & 125 & 10.6 & 20 & 3.14 & 9.14 & 9.59 \\
\hline Ho & 1.82 & 1.89 & 3.99 & 3.57 & 2.18 & 231 & 9.99 & 2.39 & 1.99 & 9.86 & 1.16 & 2.59 & 1.74 \\
\hline$E_{T}$ & 5.29 & 5.41 & 127 & 11.0 & 6.56 & 6.54 & 10.5 & 7.96 & 5.85 & 11.7 & 300 & 4.12 & 5.19 \\
\hline$T_{m}$ & 0.78 & 081 & 2.05 & 1.72 & 1.02 & 0.94 & 1.75 & 1.12 & 0.91 & 1.86 & 04 & 0.57 & 0.82 \\
\hline$Y b$ & 4.79 & 5.14 & 13.1 & 10.7 & 6.46 & 5.55 & 112 & 7.32 & 5.72 & 11.6 & 2.25 & 332 & 523 \\
\hline Lu & 068 & 0.74 & 1.89 & 1.49 & 0.91 & 0.78 & 1.62 & 104 & - 84 & 1.69 & 03 & 0.45 & 0.74 \\
\hline $\mathrm{U}$ & 746 & 88 & 20.7 & 10.1 & 9.98 & 6.95 & 12.1 & 11.9 & 7.17 & 128 & 7.89 & 6.93 & 62 \\
\hline$T_{h}$ & 428 & 50.1 & 77.1 & 39.9 & 33.6 & 57.8 & 59 & 70.1 & 28.4 & 78.6 & 27.3 & 28.1 & 30.1 \\
\hline$Y$ & 542 & 56.9 & 130 & 119 & 69.9 & 68 & 125 & 81.2 & 57.9 & 118 & 95.5 & 593 & 542 \\
\hline $\mathrm{NB}$ & 25.2 & 27 & 68.3 & 42.2 & 51.9 & 34.8 & 39.9 & 40.6 & 34.9 & 61.7 & 25.2 & 20.2 & 93.1 \\
\hline $2 \mathrm{r}$ & 397 & 303 & 168 & 157 & 255 & 264 & 183 & 192 & 248 & 188 & 195 & 124 & 191 \\
\hline $\mathrm{Hf}$ & 9.2 & 84 & 74 & 5.6 & 7.1 & 83 & 63 & 69 & 6.9 & 7.4 & 5.1 & 3.7 & 5.6 \\
\hline $\mathrm{Ta}_{\mathrm{a}}$ & 2.37 & 2.78 & 7.86 & 6.19 & 504 & 294 & 5.92 & 549 & 43 & 9.2 & 3.27 & 205 & 499 \\
\hline $\mathrm{Pb}$ & 30 & 24 & 34 & 27 & 17 & 34 & 41 & 35 & 32 & 37 & 35 & 93 & 30 \\
\hline $\mathrm{Ga}$ & 22 & 23 & 26 & 21 & 22 & 25 & 25 & 22 & 21 & 24 & 21 & 22 & 24 \\
\hline Mo & 18 & 9 & 14 & 12 & 16 & 11 & 18 & 16 & 6 & 8 & 8 & 12 & 16 \\
\hline$S_{n}$ & 8 & 10 & 4 & 3 & 7 & 6 & 12 & 12 & 9 & 8 & 11 & 7 & 16 \\
\hline w & 9.5 & 4.7 & 6.5 & 11.5 & 3.1 & 3.1 & 3.0 & 7.8 & 1.7 & 7.7 & 33 & 5.6 & 4.7 \\
\hline $\mathrm{E} \cup / \mathrm{Fu}^{\mathrm{*}}$ & 032 & 0.93 & 0.05 & 0.08 & 0.49 & 0.17 & 0.11 & 0.1 & 034 & 0.08 & 0.41 & 0.23 & 0.4 \\
\hline$M$ & 1.50 & 1.98 & 1.46 & 139 & 201 & 238 & 1.42 & 238 & 145 & 149 & 136 & 131 & 1.92 \\
\hline$T_{z x}\left({ }^{\circ} \mathrm{C}\right)$ & 847 & 899 & 792 & 790 & & 833 & 797 & 803 & 821 & 798 & 806 & 771 & 807 \\
\hline
\end{tabular}

Major element oxides and trace elements were analysed by ACM.ABSCa nada (analytical details are found in electronic Appendix $\mathrm{A}$ ). Total iron as Fe $\mathrm{O}_{3}$; major element oxides in wL \%, trace elements im ppm Abbreviations: PG: Porphyritic granite; EG: Equigranulargranite; LFP la Florida pluton; SVD: Sub-volonic dyke; FMPG= Fine matrixporphyritic granite; Ap: Aplite; CMPG: Coarse-grained matrix porphyritic granite. LFB =lachlan Fold Belt (Australia), KR=Kokshaal Range (Kyrgyzstan). $n=a v e r a g e ~ s a m p l e s, n r=n o t$ reported.

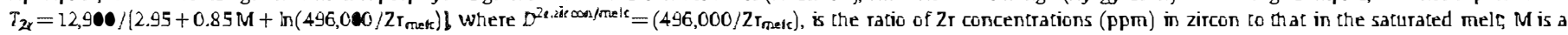
compositionalf actor that accounts for dependence of zircon solubility on SiOz and peraluminosity of the melt $\left[\left(\mathrm{Na}+K+2 \cdot \mathrm{Ca}_{\mathrm{a}}\right) / \mathrm{A} \mid\right.$. Si), all in cation fraction $]$ The geo thermometer is calibrated for $M=0.9$ to 1.7. Equation and $\mathrm{Zr}$ concentrations ( $\mathrm{ppm}$ ) in zircon ( $=496,000 \mathrm{ppm}$ ) from Miller et al. (2009).

a The LFB average was taken from Chappell and White (1992).

- The KR average was calculated ising the data shown in Table I from Konopelkn et al. (2007), using samples of the Uch-Koshkon felsic granite.

\subsection{Trace element}

The concentration ranges for the High Field Strength Elements (HSFE), such as $\mathrm{Y}, \mathrm{Nb}, \mathrm{Ga}, \mathrm{Ta}, \mathrm{U}, \mathrm{Th}$ etc, are comparable to those reported in anorogenic granitic complexes such as the A-type granites of the Lachlan Fold Belt in Australia (data in Table 1 from Chappell and White, 1992) or the Hercynian A-type granites of the Kolshaal range in Kyrgyzstan (data in Table 1 from Konopelko et al., 2007). The 


\begin{tabular}{|c|c|c|c|c|c|c|c|c|c|c|c|c|}
\hline \multirow[b]{2}{*}{$P G$} & \multirow[b]{2}{*}{$A P$} & \multirow[b]{2}{*}{ SVD } & \multicolumn{6}{|l|}{ San $B 1$ as } & \multicolumn{2}{|c|}{ Ia Chinchilla stock } & \multirow{5}{*}{$\begin{array}{l}\text { LFB }^{3} \text { Pluton } \\
\text { Av. } n=43\end{array}$} & \multirow{5}{*}{$\begin{array}{l}3 k^{x} \\
\text { Av. } n=5\end{array}$} \\
\hline & & & $P G$ & CMPG & FMPG & $P G$ & $A P$ & SVD & EG & EG & & \\
\hline $29^{\circ} 11^{\prime} 48^{\prime \prime}$ & $29^{\circ} 08^{\prime} 02^{\prime \prime}$ & $29^{\prime} 08^{\prime} 02^{\prime \prime}$ & $28^{\circ} 32^{\prime} 31^{\prime \prime}$ & $28^{\circ} 27^{\prime} 21^{\prime \prime}$ & $28^{\circ} 27^{\prime} 14^{\prime \prime}$ & $28^{\circ} 29^{\prime} 11^{\prime \prime}$ & $28^{\circ} 27^{\prime} 20^{\prime \prime}$ & $28^{\circ} 27^{\prime} 56^{\prime \prime}$ & $29^{\circ} 10^{\prime} 40^{\prime \prime}$ & $29^{\circ} 10^{\prime} 21^{\prime \prime}$ & & \\
\hline $67^{\circ} 59^{\prime} 06^{\prime \prime}$ & $67^{\circ} 0011^{\prime \prime}$ & $66^{\circ} 58^{\prime} 07^{\prime \prime}$ & $67^{\circ} 09^{\prime} 11^{\prime \prime}$ & $67^{\circ} 05^{\prime} 55^{\prime \prime}$ & $67^{\circ} 0601^{\prime \prime}$ & $67^{\circ} 04^{\prime 5}=$ & $67^{\circ} 05^{\prime} 56^{\prime \prime}$ & $67^{\circ} 05^{\prime} 36^{\prime \prime}$ & $66^{\prime} 58^{\prime} 21^{\nu}$ & $66^{\circ} 58^{\prime} 20^{\prime \prime}$ & & \\
\hline HUA 13 & HUA 5 & CHI 19 & SBP 15 & SBP 07 & SBP.09 & SPB 10 & SBP 08 & $\operatorname{SBP} 12$ & C.HI 15 & CHI 18 & & \\
\hline 72.39 & 73.28 & 57.07 & 73.92 & 76.37 & 71.83 & 76.01 & 76.24 & 54.11 & 74.54 & 75.33 & 73.83 & 73.72 \\
\hline 0.19 & 0.06 & 1.75 & 0.23 & 0.13 & 0.38 & 0.11 & 0.02 & 1.9 & 0.05 & 0.05 & 0.28 & 0.16 \\
\hline 13.11 & 13.45 & 15.26 & 12.84 & 11.85 & $13.5 \%$ & 11.99 & 13.22 & 15.02 & 12.26 & 12.79 & 12.79 & 13.45 \\
\hline 2.02 & 1.31 & 8.72 & 2.39 & 1.79 & 3.09 & 1.8 & 1.03 & 10.09 & 1.52 & 1.49 & 0.84 & 0 \\
\hline 0.03 & 0.07 & 0.14 & 0.04 & 0.03 & 0.05 & 0.03 & 0.05 & 0.16 & nd & nd & 1.54 & 1.76 \\
\hline 0.16 & 0.07 & 3.57 & 0.17 & $0.0 \%$ & 0.33 & 0.03 & 0.00 & 3.94 & 0.06 & 0.06 & 0.05 & 0.04 \\
\hline 0.97 & 0.31 & 4.81 & 0.8 & 0.68 & 1.15 & 0.66 & 0.46 & 5.13 & 0.08 & 0.05 & 0.29 & 0.16 \\
\hline 2.79 & 3.42 & 3.93 & 3.2 & 2.91 & 3.29 & 3.05 & 4.35 & 3.76 & 0.62 & 0.65 & 1.04 & 1.01 \\
\hline 5.66 & 4.75 & 3.05 & 5.5 & 5.16 & 5.39 & 5.29 & 4.1 & 3.21 & 3.74 & 3.8 & 3.42 & 3.27 \\
\hline 0.11 & 0.13 & 0.74 & 0.11 & 0.05 & 0.21 & 0.04 & 0.02 & 0.86 & 4.2 & 4.47 & 4.67 & $\square$ \\
\hline 1.65 & 1.81 & 1.49 & 0.55 & 0.69 & 0.7 & 0.64 & 0.49 & 1.66 & 0.02 & 0.02 & 0.07 & 0.05 \\
\hline \multirow[t]{2}{*}{99.08} & 98.65 & 100.53 & 99.75 & 49.73 & 노녁녀ㄴㅓㅓ & 34.64 & 9.9.98 & 99.84 & 1.81 & 1.8 & $\mathrm{nr}$ & 0.92 \\
\hline & & & & & & & & & 98.9 & 100.51 & 98.82 & 99.53 \\
\hline 6 & 8 & 6 & 8 & 11 & 16 & 9 & 23 & 7 & 7 & 21 & & \\
\hline 4 & 3 & 13 & 5 & 5 & 5 & 3 & 1 & 16 & 3 & 3 & & \\
\hline 5 & 8 & 116 & 7 & 8 & 13 & 6 & 20 & 119 & 6 & 43 & & \\
\hline 110 & 100 & 100 & 40 & $<20$ & $<20$ & 30 & 30 & 50 & 150 & 170 & & \\
\hline 1 & $<1$ & 23 & $\vdots$ & $<1$ & 2 & $<1$ & $<1$ & 21 & ] & $<1$ & & \\
\hline 10 & $<10$ & 20 & $<10$ & 20 & $<10$ & 20 & $<10$ & 20 & 70 & 20 & & \\
\hline 40 & 40 & 80 & 50 & 50 & 40 & 30 & $<30$ & 90 & 40 & 30 & & \\
\hline 26.3 & 34 & 77.4 & 15.3 & 38.8 & 31.1 & 20.5 & 140 & 36.6 & 23.8 & 22 & & \\
\hline 382 & 559 & 192 & 453 & 482 & 470 & 568 & 1000 & 255 & 684 & $68 \%$ & & \\
\hline 64 & 19 & 490 & 45 & 31 & 63 & 16 & 4 & 419 & 8 & !! & & \\
\hline 233 & 105 & 244 & 108 & 79 & 155 & 45 & 10 & 314 & 16 & 25 & & \\
\hline 49.3 & 11 & 32.9 & 75.3 & 64.2 & 52.1 & 72 & 13 & 45.5 & 15.2 & 17.8 & & \\
\hline 112 & 25.9 & 72.6 & 165 & 142 & 117 & 170 & 38.1 & 102 & 41.9 & 49.7 & 130 & 100 \\
\hline 12.5 & 2.92 & 8.34 & 18.1 & 15.9 & 13.6 & 20 & 5.3 & 12.3 & 5.63 & 6.47 & & \\
\hline 44.8 & 11.9 & 33.4 & 59.5 & 53.5 & 47.5 & 73.9 & 26.0 & 48.0 & 29.5 & 313 & & \\
\hline 9.24 & 2.67 & 6.96 & 11.1 & 10.4 & 9.58 & 16.8 & 7.95 & 9.87 & 8.55 & 9.55 & & \\
\hline 1.23 & 0.27 & 2.09 & 0.65 & 0.5 & 0.9 & 0.43 & 0.06 & 2.3 & 0.17 & 0.19 & & \\
\hline 7.01 & 2.55 & 5.75 & 8.86 & 8.55 & 8.21 & 15.3 & 8.42 & 8.89 & 9.23 & 10.6 & & \\
\hline 1.26 & 0.68 & 0.97 & 1.48 & 1.48 & $\square$ & 3.03 & 1.99 & 1.43 & 2.27 & 2.67 & & \\
\hline 6.92 & 4.76 & 5.37 & 8.19 & 8.43 & 8.04 & 19 & 13.9 & 7.95 & 16 & 18.3 & & \\
\hline 1.2 & 0.96 & 0.93 & 1.5 & 1.57 & 1.46 & 3.72 & 2.91 & 1.48 & 3.24 & 3.73 & & \\
\hline 3.48 & 3.03 & 2.52 & 4.32 & 4.58 & 4.22 & 11.4 & 9.99 & 4.26 & 10.6 & 11.6 & & \\
\hline 0.55 & 0.59 & 0.35 & 0.62 & 0.70 & 0.66 & 1.88 & 2.01 & 0.65 & 1.84 & 2.04 & & \\
\hline 3.62 & 4.22 & 2.17 & 3.7 & 4.22 & 4.2 & 11.6 & 14.1 & 3.98 & 12.4 & 13.2 & & \\
\hline 0.52 & 0.61 & 0.32 & 0.5 & 0.6 & 0.59 & 1.59 & 2.07 & 0.54 & 1.82 & 1.88 & & \\
\hline 5.93 & 9.06 & 7.58 & 12.1 & 8.83 & 6.04 & 10.3 & 28.3 & 6.09 & 33.3 & 34.4 & D & 10 \\
\hline 28.2 & 9.17 & 5.76 & 73.4 & 82.7 & 46.1 & 86.3 & 31.9 & 17.5 & 35.9 & 43.3 & 24 & 51 \\
\hline 36.5 & 32.1 & 27.4 & 45.1 & 45.1 & 45.8 & 113 & $: 03$ & 44.2 & 125 & 142 & 71 & 65 \\
\hline 23 & 33.1 & 33.9 & 33.6 & 33.3 & 37.3 & 81.1 & 45.7 & 46.5 & 54.5 & 58.2 & 26 & 26 \\
\hline 156 & 36 & 238 & 234 & 161 & 261 & 208 & 81 & 247 & 104 & 104 & 322 & 185 \\
\hline 4.6 & 1.7 & 5.4 & 7.1 & 5.9 & 7.5 & 9.2 & 6.4 & 6.5 & 5.7 & 5.7 & & \\
\hline 2.84 & 5.87 & 4.18 & 3.15 & 4.94 & 6.08 & 6.59 & 22.8 & 4.04 & 13.8 & 15.0 & & \\
\hline 29 & 28 & 10 & 29 & 25 & 17 & 29 & 28 & 10 & 36 & 33 & & \\
\hline 22 & 26 & 21 & 24 & 21 & 23 & 30 & 34 & 21 & 29 & 29 & 22 & 24 \\
\hline 14 & 3 & 5 & $<2$ & $<2$ & $<2$ & 2 & $<2$ & 3 & 14 & 16 & & \\
\hline 7 & 22 & 10 & 9 & 8 & 12 & 6 & 16 & 6 & 41 & 41 & & \\
\hline 4.1 & 16.3 & 5.7 & 24 & 10.5 & 12.1 & 5.4 & 7.8 & 2.8 & 6.4 & 5.0 & & \\
\hline 0.47 & 0.32 & 1.02 & 0.2 & 0.16 & 0.31 & 0.08 & 0.02 & 0.75 & 0.06 & 0.06 & & \\
\hline 1.36 & 1.19 & 2.25 & 1.41 & 1.35 & 1.45 & 1.38 & 1.32 & 2.44 & 1.34 & 1.34 & & \\
\hline 787 & 683 & & 819 & & 827 & 811 & 735 & & 754 & 754 & & \\
\hline
\end{tabular}

granitoids also plot in the field of A-type granites on the discrimination diagrams of Whaten et al. (1987) (Fig. 7). Primitive mantlenormalized spider disgrams show marked negative $\mathrm{Ba}, \mathrm{Nb}, \mathrm{Sr}, \mathrm{P}$ (with the exception of the Huaco granitic complex which only shows a moderate negative anomaly), Fu and $\mathrm{Ti}$ anomalies and significant enrichment in Rb, Th, U, and $\mathrm{Pb}$ (Fig. 8). Rare earth element (RFE) abundances vary from 116 to $554 \mathrm{ppm}$. The RFE patterns are flat or slightly light RFE enriched (LREE) with significant negative Fu 
Table 2

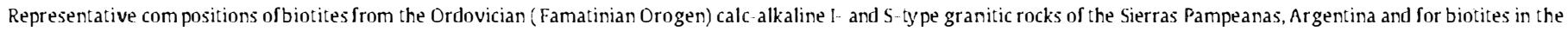
Carboniferous granites.

\begin{tabular}{|c|c|c|c|c|c|c|c|c|}
\hline \multirow{2}{*}{$\frac{\text { Rock type }}{\text { Sample }}$} & & I-type & & \multicolumn{2}{|c|}{ 1-type } & & & S-type \\
\hline & & \multicolumn{2}{|l|}{ ASP- $: 20$} & \multicolumn{3}{|c|}{ ASP-120/1 } & & 32-JEOL-00 \\
\hline Lithology & & \multicolumn{2}{|l|}{ Hbl-Bt Gde } & \multicolumn{3}{|c|}{ Hbl-Bt Gde } & & Crd- Qtz-rich Gtoid \\
\hline \multicolumn{9}{|l|}{ wt.. } \\
\hline $\mathrm{SiO}_{2}$ & & \multicolumn{2}{|l|}{35.53} & \multicolumn{3}{|c|}{35.68} & & 35.59 \\
\hline $\mathrm{TiO}_{2}$ & & \multicolumn{2}{|l|}{2.83} & & 78 & & & 2.49 \\
\hline $\mathrm{Al}_{2} \mathrm{O}_{3}$ & & 16.38 & & & & & & 19.99 \\
\hline $\mathrm{FeO}$ & & 18.68 & & & & & & 17.12 \\
\hline $\mathrm{MnO}$ & & 0.35 & & & 32 & & & 0.58 \\
\hline $\mathrm{MgO}$ & & 10.52 & & & & & & 9.8 \\
\hline $\mathrm{CaO}$ & & 0.03 & & & 02 & & & 0.02 \\
\hline $\mathrm{Na}_{2} \mathrm{O}$ & & 0.14 & & & 09 & & & 0.22 \\
\hline $\mathrm{K}_{2} \mathrm{O}$ & & 9.7 & & & & & & 9.52 \\
\hline$F$ & & 0.16 & & & 01 & & & 0.23 \\
\hline $\mathrm{Cl}$ & & 0.04 & & & 05 & & & 0.04 \\
\hline Total & & 94.36 & & & & & & 95.65 \\
\hline O_F_Cl & & 0.08 & & & 02 & & & 0.11 \\
\hline CTotal & & 94.28 & & & & & & 95.54 \\
\hline $\mathrm{FeO}^{\prime} / \mathrm{MgO}$ & & 1.78 & & & 88 & & & 1.75 \\
\hline $\mathrm{T}\left({ }^{\circ} \mathrm{C}\right)$ & & 800 & & 80 & & & & 800 \\
\hline$F_{\text {mek }}(p p m)$ & & 12 & & 16 & & & & 468 \\
\hline$C l_{\text {mekr }}(\mathrm{ppm})$ & & 156 & & 9 & & & & 0 \\
\hline Sample & FiA17/2 & $\mathrm{F}[\mathrm{A} 17 / 5$ & FIA17/6 & & 2AP33/4 & & $2 A P 33 / 49$ & $2 A P 33 / 51$ \\
\hline wt. & & & & & & & & \\
\hline $\mathrm{SiO}_{2}$ & 36.02 & 36.18 & 35.45 & & 34.41 & & 34.35 & 34.40 \\
\hline $\mathrm{TiO}_{2}$ & 2.09 & 1.57 & 1.47 & & 3.09 & & 2.95 & 3.11 \\
\hline $\mathrm{Al}_{2} \mathrm{O}_{3}$ & 18.90 & 19.63 & 20.32 & & 15.01 & & 15.87 & 14.94 \\
\hline FeO & 27.55 & 27.83 & 27.36 & & 29.24 & & 27.75 & 28.54 \\
\hline $\mathrm{MnO}$ & 0.35 & 0.38 & 0.32 & & 0.49 & & 0.35 & 0.44 \\
\hline $\mathrm{MgO}$ & 0.72 & 0.71 & 0.65 & & 3.34 & & 3.21 & 3.68 \\
\hline $\mathrm{CaO}$ & 0.00 & 0.06 & 0.03 & & 0.01 & & 0.00 & 0.00 \\
\hline $\mathrm{Na}_{2} \mathrm{O}$ & 0.13 & 0.13 & 0.13 & & 0.08 & & 0.06 & 0.06 \\
\hline $\mathrm{K}_{2} \mathrm{O}$ & 9.46 & 9.33 & 9.34 & & 9.37 & & 9.42 & 9.38 \\
\hline$F$ & 1.15 & 1.29 & 1.05 & & 0.25 & & 0.24 & 0.47 \\
\hline $\mathrm{Cl}$ & 0.39 & 0.40 & 0.40 & & 0.25 & & 0.21 & 0.28 \\
\hline Total & $96.8 ?$ & 97.52 & 96.52 & & 95.56 & & 94.40 & 95.29 \\
\hline O_F_Cl & 0.57 & 0.63 & 0.53 & & 0.16 & & 0.15 & 0.26 \\
\hline CTotal & 96.24 & 96.89 & 95.99 & & 95.40 & & 94.25 & 95.03 \\
\hline $\mathrm{FeO} / \mathrm{MgO}$ & 38.26 & 39.20 & 42.09 & & 8.75 & & 8.64 & 7.76 \\
\hline$T\left({ }^{\circ} \mathrm{C}\right)$ & 800 & 800 & 800 & & 800 & & 800 & 800 \\
\hline$F_{\text {mekr }}(p p m)$ & 4430 & 3860 & 2860 & & 590 & & 910 & 1180 \\
\hline $\mathrm{Cl}_{\text {mell }}(\mathrm{ppm})$ & 1050 & 960 & 120 & & 520 & & 630 & 730 \\
\hline Sample & HUA12/10 & HUA12/9 & SBP2/1 & SBP2/3 & & SBP3/3 & TUY12/2 & TUY 2/3 \\
\hline wt. & & & & & & & & \\
\hline $\mathrm{SiO}_{2}$ & 34.47 & 36.13 & 34.66 & 33.85 & & 35.03 & 34.90 & 34.03 \\
\hline $\mathrm{TiO}_{2}$ & 2.10 & 2.40 & 2.08 & 2.53 & & 2.10 & 3.08 & 2.93 \\
\hline $\mathrm{Al}_{2} \mathrm{O}_{3}$ & 29.03 & 18.93 & 18.26 & 18.21 & & 18.97 & 18.79 & 18.99 \\
\hline FeO & 26.35 & 25.31 & 29.39 & 30.12 & & 27.69 & 27.16 & 26.88 \\
\hline $\mathrm{MnO}$ & 0.57 & 0.71 & 0.61 & 0.52 & & 0.65 & 0.52 & 0.39 \\
\hline $\mathrm{MgO}$ & 2.28 & 2.57 & 0.63 & 0.49 & & 0.77 & 2.56 & 2.55 \\
\hline $\mathrm{CaO}$ & 0.00 & 0.01 & 0.09 & 0.15 & & 0.07 & 0.00 & 0.09 \\
\hline $\mathrm{Na}_{2} \mathrm{O}$ & 0.08 & 0.10 & 0.09 & 0.12 & & 0.13 & 0.12 & 0.10 \\
\hline $\mathrm{K}_{2} \mathrm{O}$ & 9.58 & 9.75 & 9.02 & 9.16 & & 9.16 & 9.77 & 9.04 \\
\hline F & 0.60 & 1.07 & 1.43 & 0.98 & & 1.25 & 0.49 & 0.14 \\
\hline $\mathrm{Cl}$ & 0.07 & 0.02 & 0.25 & 0.31 & & 0.30 & 0.10 & 0.15 \\
\hline Total & 95.12 & 97.00 & 96.51 & 96.44 & & 96.12 & 97.49 & 95.29 \\
\hline $\mathrm{O}_{-} \mathrm{F} \mathrm{Cl}$ & 0.27 & 0.46 & 0.66 & 0.48 & & 0.59 & 0.23 & 0.09 \\
\hline CTotal & 94.85 & 96.54 & 95.85 & 95.96 & & 95.53 & 97.26 & 95.2 \\
\hline $\mathrm{FeO} / \mathrm{MgO}$ & 11.56 & 9.85 & 46.65 & 61.47 & & 35.96 & 10.61 & 10.54 \\
\hline$T\left({ }^{\circ} \mathrm{C}\right)$ & 800 & 800 & 800 & 800 & & 800 & 800 & 800 \\
\hline$F_{\text {mek }}(P p m)$ & $56: 0$ & 1880 & 4440 & 4750 & & 4860 & 360 & 350 \\
\hline$C l_{\text {melt }}(p p m)$ & 650 & 90 & 830 & 840 & & 740 & 800 & 260 \\
\hline
\end{tabular}

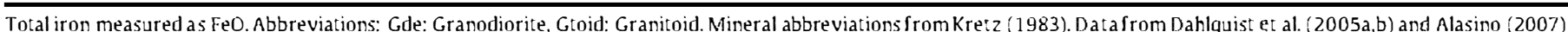
Calculated F and C following Muñoz (1984, 1992). Structural formulae for biotites and calculated $\mathrm{F}_{\text {mek }}$ and $\mathrm{Cl}_{\text {mek }}$ are found in the supplemental electronic Table.

identical to those of the MLinoz (1992) model, but for F there is a significant divergence in the calculated values which increase with decreasing $\mathrm{mg} \#$ (increasing Fe content of the biotice). For example, for sample SBP-2/1 the Muñoz (1992) medel gives a calculated $F$ value of
5800 ppm while the Icenhower and London (1997) model gives a calculated $F$ value of $42,200 \mathrm{ppm}$. It should be noted that the Icenhower and London (1997) model was not calibrated for low mg\# (high Fe) biotites and values derived from this model for very 

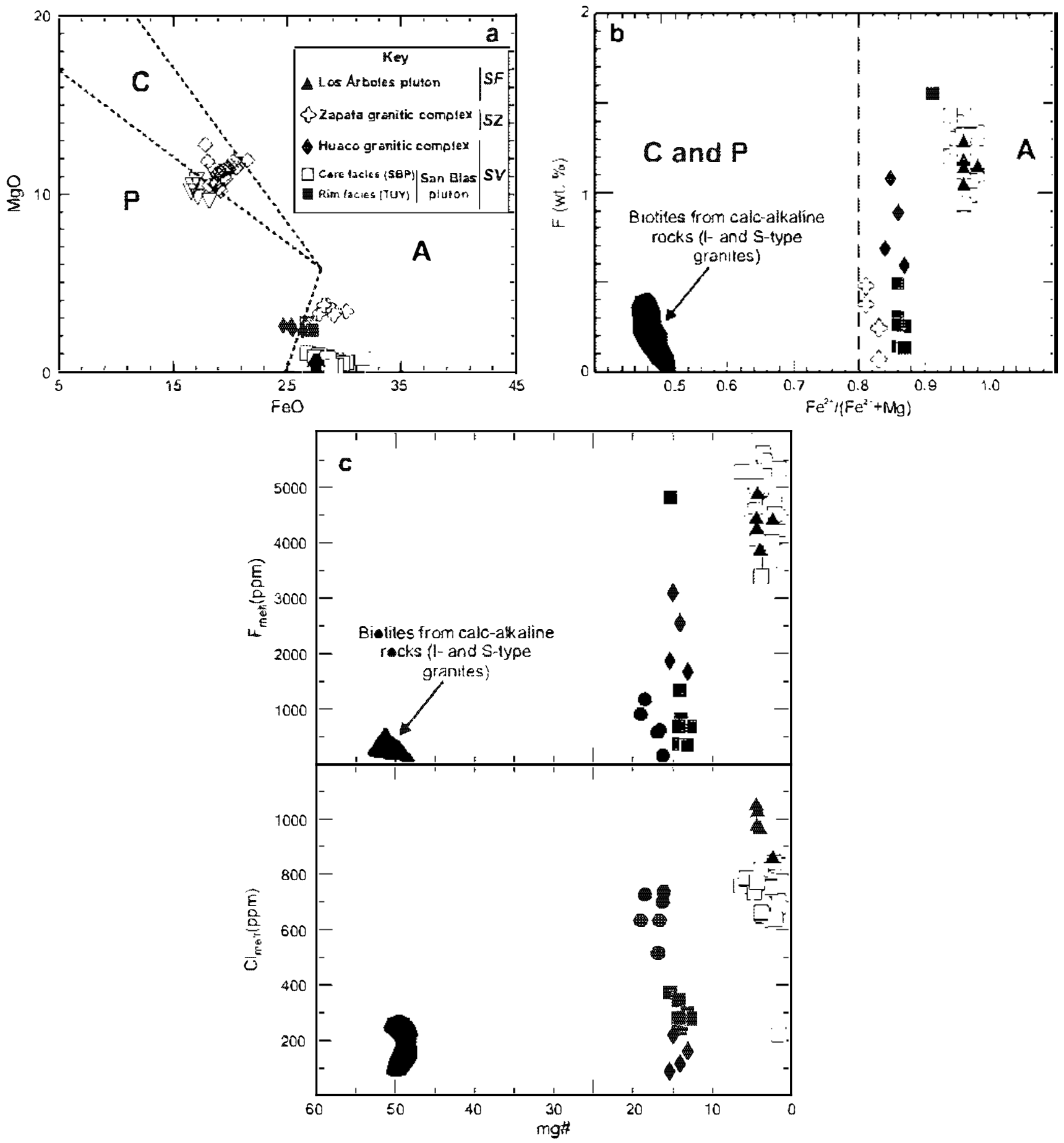

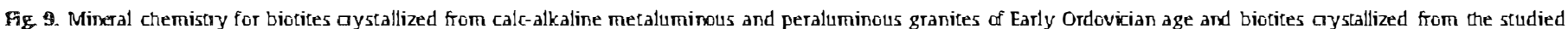

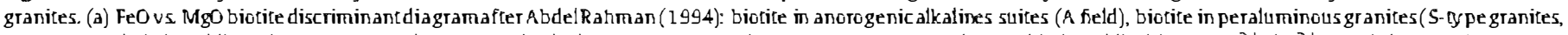

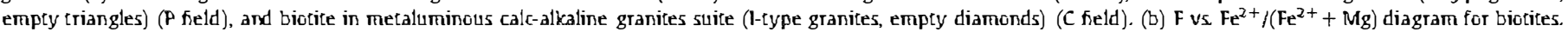

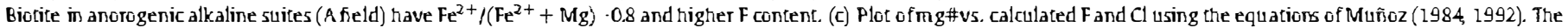

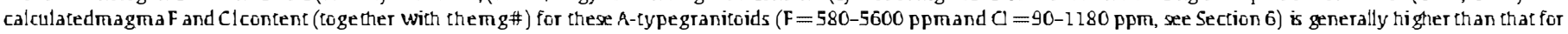

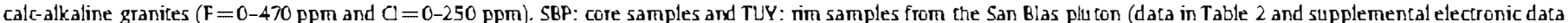
table).

iron-rich biotites range up to $10 \mathrm{wt} . \% \mathrm{~F}$ in the melt, an unrealisucally high-F content. The calculated $F$ values reported here are based on the Muñoz (1992) model.

A component of the definition of A-type grames is that the melt contains relatively high $F$, but this is rarely quantified. In this paper we present one of the first attempts to provide quantirarive data on magma $\mathrm{F}$ (and $\mathrm{Cl}$ ) content. Biotites in the Eally Ordovician granitic rocks with calc-alkaline signature (formed dunng the Fanatinian orogen, NW Argentina, Dahlquist et al., 2005a, 2008) have mg $~ 50$ and the magmas are characterized by both low $F(0$ to $470 \mathrm{ppm}$ ) and $\mathrm{Cl}(0$ to $250 \mathrm{ppm})$. For the A-type granicoids, biotite mg \#s vary from 19 to 2, and for each pluton or complex there is a restricted range in the $\mathrm{mg}$ \# of the biotites and the magna $\mathrm{F}$ and $\mathrm{Cl}$ content (Table 2 and supplemental electronic data table, and Fig. 9c). The Los Árboles (F-3850 to $4900 \mathrm{ppm}, \mathrm{Cl}-860$ to $1050 \mathrm{ppm}$ ) and San Blas (F $=3400$ to $5600 \mathrm{ppm}, \mathrm{Cl}-640$ to $850 \mathrm{ppm}$ ) plutons have the 
Table 3a

Rb-Sr composition of representative samples from the studied granitic rocks.

\begin{tabular}{|c|c|c|c|c|c|c|}
\hline Samples & $\mathrm{SiO}_{2}$ (wt.o) & Ro & $S r$ & ${ }^{86} \mathrm{Rb} /{ }^{87} \mathrm{Sr}$ & $\left.\left({ }^{87} \overline{S r}\right)^{\beta t} \overline{S r}\right)_{h d a d y}$ & $\left({ }^{87} \mathrm{Sr} /{ }^{86} \mathrm{Sr}\right) t$ \\
\hline \multicolumn{7}{|c|}{$t=323 \mathrm{Ma}$} \\
\hline 2AP-26 & 32.30 & 438.1 & 53.1 & 24.1347 & 0.820759 & 0.309805 \\
\hline 2AP-27 & 75.11 & 506.8 & 19.3 & 78.4302 & 1.038146 & 0.677580 \\
\hline 2AP-33 & 31.86 & 378.9 & 73.1 & 15.1039 & 0.780833 & 0.711396 \\
\hline 2AP- 35 & 73.37 & 313.7 & 44.1 & 20.7779 & 0.805605 & 0.71083 \\
\hline \multicolumn{7}{|c|}{$t=354 \mathrm{Ma}$} \\
\hline HUA-4 & 74.61 & 380.6 & 64.2 & 17.2930 & 0.791567 & 0.304417 \\
\hline HUA-6 & 32.79 & 390.6 & 57.0 & 20.0153 & 0.805095 & 0.704225 \\
\hline HUA-13 & 72.39 & 392.8 & 30.5 & 37.8985 & 0.882489 & 0.691495 \\
\hline HUA-14 & 70.86 & 365.0 & 66.4 & 16.0275 & 0.786958 & 0.706185 \\
\hline
\end{tabular}

The decay constants used in the calculations are $\lambda^{47} R^{\prime}, \quad .42 \times 10^{-11}$ nnd $\mathrm{A}^{147} \mathrm{sm}$ $6.54 \times 10^{-12}$ year $^{-1}$ recommended by the IUGS Sub-commission for Geochronology (Steiger and Jäger, 1977). Epsilon-Sr ; sir values were calculated relative

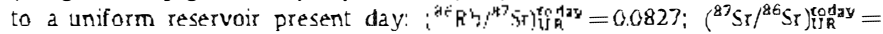
0.3045 F.pon $\mathrm{Nd}\left(\varepsilon_{\mathrm{N} a}\right)$ values were calculated relative to a chondrite present day:

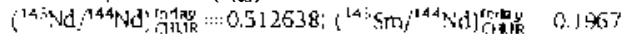

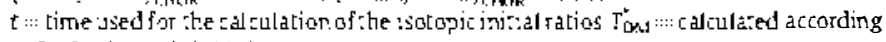
to De Paolo et al. (1991).

The ages are: FA-1) (335 Ma, Grissom et al., 1998), HUA-4 (354 Ma. Sölner et al., 2007), SBP-15 (340Ma, Dahlquist et al, 2006), ZAP (323Ma, McBride, 1972; McBride e[ al., 1976).

highest calculated magma $\mathrm{F}$ and $\mathrm{Cl}$ contents. The Huaco granitic complex ( $\mathrm{F}=1700$ to $3100 \mathrm{ppm}, \mathrm{Cl}=90$ to $220 \mathrm{ppm}$ ) has highe: calculated magma $\mathrm{F}$ content and lower calculated magma $\mathrm{C}$ content than the Zapata granitic complex (F=580 to $1180 \mathrm{ppm}, \mathrm{Cl}=510$ to $740 \mathrm{ppm}$ ). It should be noted that while the calculated magma $F$ and $\mathrm{Cl}$ content for these A-type granitoids is generally higher than that for calc-alkaline granites, the values are not excessively high, i.e., water is still a significant part of the volatile component of the magmas.

\section{Sr and Nd isotope systematics}

\section{1. $R b-S r$ isotopes}

Initial ${ }^{87} \mathrm{Sr} /{ }^{86} \mathrm{Sr}$ ratios of ca. 0.7088 (for a U-Pb SHRIMP zircon crystallization age of $340 \pm 3 \mathrm{Ma}$ ) were reported by Dahlquist et al. (2006) for the San Blas pluton. This isotopic ratio is indistinguishable from the initial ${ }^{87} \mathrm{Sr} /{ }^{86} \mathrm{Sr}$ ratio $(0.7086)$ of the Capilla del Monte granite in the Sierra de Córdoba (Fig. 1), which is also Early Carboniferous (Rb-Sr isochron age of $337 \mathrm{Ma}$; Saavedra et al., 1998). This similarity suggests that the magmas for these two plutons came from an isotopically similar source.

New whole-rock Rb-Sr isotope data for the Zapata and Huaco granitic complexes are reported in Table 3a. Analytical details are found in electronic Appeadix A. For the Zapata granilic co:nplex initial ratios at the reference age of $323 \mathrm{Ma}$ range from 0.7098 to 0.7114 . A fourth sample (ZAP-27) yielded an anomalous value of 0.6776 . Because the reference age is a $\mathrm{K}-\mathrm{Ar}$ cooling age the initial $\mathrm{Sr}$ isotope ratios of the Zapata magmas were probably lower than the values given above. For the Huaco granitic complex, initial ratios at a reference age of $354 \mathrm{Ma}$ for three of the samples range from 0.7044 to 0.7062 . Again, a fourth sample (HUA-13) y1e.ded an anomalous initia! ratio of 0.6915 . The two anomalous samples also show lower $\mathrm{Sr}$ contents, probably retlecting perrurbation of the Rb-Sr systematics after crystallization by an alteration event. Isochron plots for both sets of samples yield errorchrons. In part:cular, Söllner et al. (2007) recngn:zed an alteration event in the Huaco gran'e at $315 \pm 42 \mathrm{Ma}$. Well constrained Sr isotope initial ratios for the Carboniferous granites are highly variable from 0.7044 to 0.7086 .

\subsection{Sm-Nd isotopes}

Nineteen samples from the Los Árboles $(n=5)$ and the San Blas $(n=4)$ plutons, and the Huaco $(n=5)$ and the Zapata $(n=5)$ granitic complexes were analysed for their $\mathrm{Nd}$ isotopic composition. Aari'y iral details are found in electronic Appendix A. Fpsilnn Nd values ( $\varepsilon_{\mathrm{Ndt}}$ ) were calculated for the inferred crystallization age (Table 3b).

All samples have roughly similar ${ }^{147} \mathrm{Sm} /{ }^{144} \mathrm{Nd}(0.1090-0.1616)$ and ${ }^{143} \mathrm{Nd} /{ }^{144} \mathrm{Nd}(0.512234-0.512537)$ ratios. Ranges of $\varepsilon_{\mathrm{Ndt}}$ values are: Los Árboles pliton $(-0.8$ to -2.6 , at $335 \mathrm{Ma}$ ), San Blas pluton $(+0.6$ to -4.8 ; at $340 \mathrm{Ma})$, Huaco granitic complex ( - . 2.4 to -3.4; at $354 \mathrm{Ma}$ ), Zapata glanit:c complex ( 2.6 to -3.9 ; at $323 \mathrm{Ma}$ ) (Table 35). Except for one sample from the San Blas pluton $\left[58 \mathrm{~F}^{*}-10\right.$, $\varepsilon_{\mathrm{Ndt}}=+0.6$, Table $3 \mathrm{~b}$ ) all the $\varepsilon_{\mathrm{Ndt}}$ s are negative and vary between -0.8 and -4.8 . Nd model ages calculated according to De Paolo et al. (1991) are between 0.96 and $1.72 \mathrm{Ga}$, i.e., Proternzoic, with a peak between 1.00 and $1.40 \mathrm{Ga}$ (Table 3b).

Table 3 b

Sm-Nd composition of representative samples from the studied granitic rocks.

\begin{tabular}{|c|c|c|c|c|c|c|c|c|c|}
\hline Samples & $\mathrm{SiO}_{2}$ (wt. & Age (Ma) & $S m$ & Nd & ${ }^{147} \mathrm{Sm} /{ }^{144} \mathrm{Nd}$ & $\left({ }^{145} \mathrm{Nd} /{ }^{144} \mathrm{Nd}\right)_{\mathrm{xday}}$ & $\left({ }^{143} \mathrm{Nd} /{ }^{144} \mathrm{Nd}\right) \mathrm{t}$ & $\varepsilon_{\text {Nar }}$ & $T_{\mathrm{DM}}^{*}(\mathrm{Gd})$ \\
\hline A $A-3$ & 69.21 & 335 & 12.1 & 67.1 & 0.1090 & 0.512351 & 0.512112 & -1.8 & 1.03 \\
\hline FIA -8 & 30.51 & 335 & 11.9 & 640 & 0.1124 & 0.512320 & 0.512073 & -2.6 & 1.10 \\
\hline 月A-1? & 7450 & 335 & 12.1 & 51.5 & 0.1420 & 0.512434 & 0.512123 & -1.6 & 1.29 \\
\hline คA -18 & 75.30 & 335 & 12.5 & 52.0 & 0.1453 & 0.512458 & 0.512139 & -1.3 & 1.30 \\
\hline FIA -22 & 32.77 & 335 & 11.2 & 61.7 & 0.1097 & 0.512406 & 0.512165 & -0.8 & 0.96 \\
\hline HUA-4 & 34.61 & 354 & 4.58 & 20.10 & 0.1377 & 0.512338 & 0.512019 & -3.2 & 1.40 \\
\hline HUA-6 & 72.79 & 354 & 10.2 & 46.5 & 0.1326 & 0.512365 & 0.512058 & -2.4 & 1.28 \\
\hline HUA-? & 72.62 & 354 & 9.75 & 43.6 & 0.1352 & 0.512335 & 0.512022 & -3.1 & 1.37 \\
\hline HUA-12 & 73.30 & 354 & 10.5 & 49.1 & 0.1293 & 0.512352 & 0.512052 & -2.5 & 1.25 \\
\hline HUA-13 & 72.39 & 354 & 9.24 & 44.8 & 0.1247 & 0.512318 & 0.512029 & -3.0 & 1.25 \\
\hline SBP-6 & 75.49 & 340 & 17.0 & 82.1 & 0.1252 & 0.512234 & 0.511955 & -4.8 & 1.38 \\
\hline SBP-9 & 31.83 & 340 & 9.58 & 47.5 & 0.1219 & 0.512395 & 0.512124 & -1.5 & 1.09 \\
\hline SBP-10 & 36.01 & 340 & 16.8 & 73.9 & 0.1374 & 0.512537 & 0.512231 & 0.6 & 1.04 \\
\hline SBP- 15 & 73.92 & 340 & 11.10 & 59.50 & 0.1128 & 0.512365 & 0.512114 & -1.3 & 1.04 \\
\hline 2AP-26 & 32.30 & 323 & 13.10 & 92.30 & 0.1120 & 0.512258 & 0.512021 & -3.9 & 1.19 \\
\hline 2AP-27 & 35.11 & 323 & 18.60 & 69.60 & 0.1616 & 0.512430 & 0.512088 & -2.6 & 1.32 \\
\hline 2AP-29 & 73.46 & 323 & 15.10 & 64.50 & 0.1415 & 0.512366 & 0.512067 & -3.0 & 1.41 \\
\hline 2AP-33 & 31.86 & 323 & 9.31 & 46.30 & 0.1268 & 0.512306 & 0.512038 & -3.6 & 1.29 \\
\hline 2AP-35 & 73.37 & 323 & 6.60 & 33.80 & 0.1180 & 0.512297 & 0.512047 & -3.4 & 1.20 \\
\hline
\end{tabular}

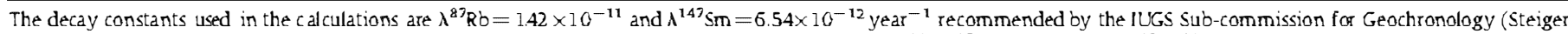

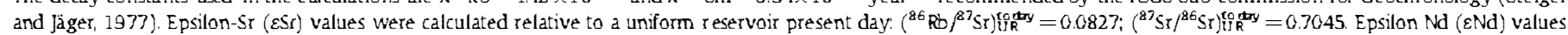

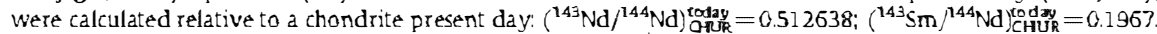

$\mathrm{t}=$ time used for the calculation of the isotopic initial ratios. $I_{\mathrm{DM}}^{*}=$ calculated according to De Paolo et al. (1991)

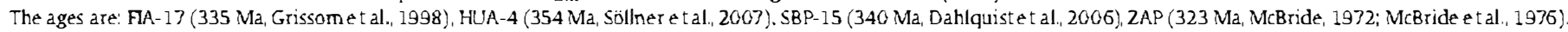




\section{Petrogenetic discussion}

The whole-rock geochemical signatures (Figs. 4, 6, 7, 8) and the biotite chemistry (Fig. 9) indicate that these Early Carboniferous granitoids are A-type granitoids. As suggested by Eby (1990, 1992). A-type granites can be formed in a variety of ways. The challenge is to determine which of the possible petrogenetic models applies to a particular occurrence. The proposed petrogenetic models are (i) fractionation of mantle-derived magma, (ii) reaction of mantle-derived magma with crustal rocks, (iii) melting of deep continental crust, and (iv) partial melting of continental crust (metasomatized or not) (Abdel Rahman, 2006; Konopelko et al., 2007). In particular, a petrogenetic model (iv); has been invoked to explait the origit of A-type felsic melts (see Abdel Rahman, 2006 and references therein).

\subsection{Source of the granitoid magmas - discrimination diagrams and isotopic data}

On the tectonic discriminant diagram of Pearce et al. (1984), the granitic rocks plot in the field of within-plate granite (WPG, Fig. 10a). Eby (1990, 1992) subdivided the A-type granires into two groups: $A_{1}$ and $A_{2}$, both emplaced in anorogetic settings. It was suggested that the $A_{1}$ group was mantle-derived while the $A_{2}$ group was derived from melting of continental crust with or without a mantle input. On the $\mathrm{Y}-\mathrm{Nb}-3 \times \mathrm{Ga}$ (Fig. 10b) discriminant diagram of Eby (1990) the granites plot in the $A_{2}$ field which is compatible with a dominantly crustal origin.

The isolopic data for the Early Carboniferous gramites (negative $\varepsilon_{\text {Ndt }}$ values and comparatively moderate to high $\mathrm{Sr}$ isotope initial ratios) strongly suggest the involvement of crustal sources in the generation of the magmas that contained $\mathrm{Nd}$ extracted from the mantle in the Mesoproterozoic (between 1.0 and $1.4 \mathrm{Ga}$ ) (Table $3 \mathrm{~b}$ ). Significant participation of Ordovician meta-granitoids in the source of the Huaco granitic complex was also recogtized by Grosse et al. (2009). Consistently, there are A-type granites that postdate spatially associated calc-alkaline rocks and that have isotopic and trace element compositions that suggest derivation from these calc-alkaline precursors eg., North America, Anderson, 1983; Egypt, Abdel Rahman, 2006; Kyrgyzstan, Konopelko et aL, 2007).

Most of the Early Ordovician granites show $T_{\mathrm{DM}}$ ages between 1.5 and $1.7 \mathrm{Ga}$ and Nd isolopic signatures $\left(\varepsilon_{\mathrm{N}, \mathrm{d}}\right.$ values between -5 and -6 at the time of crystallization between 490 and $468 \mathrm{Ma}$ ) (Dahlquist et al., 2008, and references therein). However, $\varepsilon_{\mathrm{Ndt}} \mathrm{s}$ are less negative

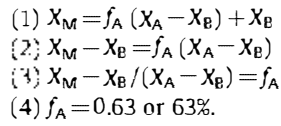

$X_{A}=$ Isotopic composition of asthenospheric mantle, ${ }^{143} \mathrm{Nd} /{ }^{144} \mathrm{Nd}=0.512203$; $X_{B}=$ Isotopic compositions of potential crustal pro:oliths that outcrop in the Sierras Pampeanas: I- and S-type Ordovician granites, ${ }^{14} \mathrm{NA} /{ }^{144} \mathrm{NA}=0.511 \mathrm{~K}^{-3}: \mathrm{X}_{\mathrm{M}}=$ Isotopic assumed mixed composition, represented by Carboniferous A-type granites, ${ }^{143} \mathrm{Nd}$ / ${ }^{144} \mathrm{Nd}=0.512078$. $f_{\mathrm{A}}=$ Asthenospheric mantle fraction in the mixture. ${ }^{143} \mathrm{Nd} /{ }^{144} \mathrm{Nd}$ compositions for $X_{A}, X_{g}$, and $X_{M}$, are recalculated to $t=338 \mathrm{Ma}$, the average of the Carboniferous crystallization ages (Table $3 \mathrm{~b}$ ). Data used in the calculation are available in the isotopic supplemental electronic data table.

( : 0.6 to -4.8 at 323 to $354 \mathrm{Ma}$ ) compared to values of Famatinian granitoids at the same time $(-4.8$ to -8.5$)$ (Table $3 \mathrm{~b}$ and in a supplemental electronic data table). The $\varepsilon_{\text {Ndt }}$ values for the Early Carboniferous granitoids (Table $3 \mathrm{~b}$ and supplemental electronic data table) are displaced towards $\varepsilon_{\text {Ndt }}$ typical of the asthenospheric mantle. Thus, the participation of an asthenospheric component (together with the old lithosphere component; is required to satisfy the $\varepsilon_{\text {Ndt }}$ values calculated for the Early Carboniferous granitoids. A simple mixing calculation using equation 9.1 of Faure (1986, page 141) and a Nd isotopic asthenospheric mantle signature (CHUR) and Nd isotopic Ordovician graniloids (potential crustal protoliths) data at the time of crystallization of the Carboniferous A-type granites $(t=338 \mathrm{Ma})$ indicates a dominant asthenospheric participation (63\% and 37\% of asthenospheric mantle and continental lithosphere, respectively: calculation in Table 4 and isotopic data can be found in a supplemental electronic data table). In fact our interpretation is that the primary magma source was an asthenospheric mantle with subsequent crustal contamination, a hypothesis that is in accord with other works (e.g., Konopelko et al., 2007; Bonin, 2007). In agreement with Bonin (2007), the original concept of A-type gratites of Loiselle and Wones (1979) contrasts sharply with the general philosophy of the overall genetic alphabet, where I-, S-, and M-type granites are derived from igneous, sedimentary and mantle source, respectively. A-type granites were defined as anorogenic granites that occur in rift zones and stable continental blocks, without mentioning a particular source. Perhaps the most important point from the abstract of Loiselle and Wones (1979) was that A-type granites have a wide range of isotopic compositions (initial ${ }^{87} \mathrm{Sr} /{ }^{86} \mathrm{Sr}$ ratios ranging from 0.703 to 0.712), sloggesting different mixing proportions of asthenospheric

a

b

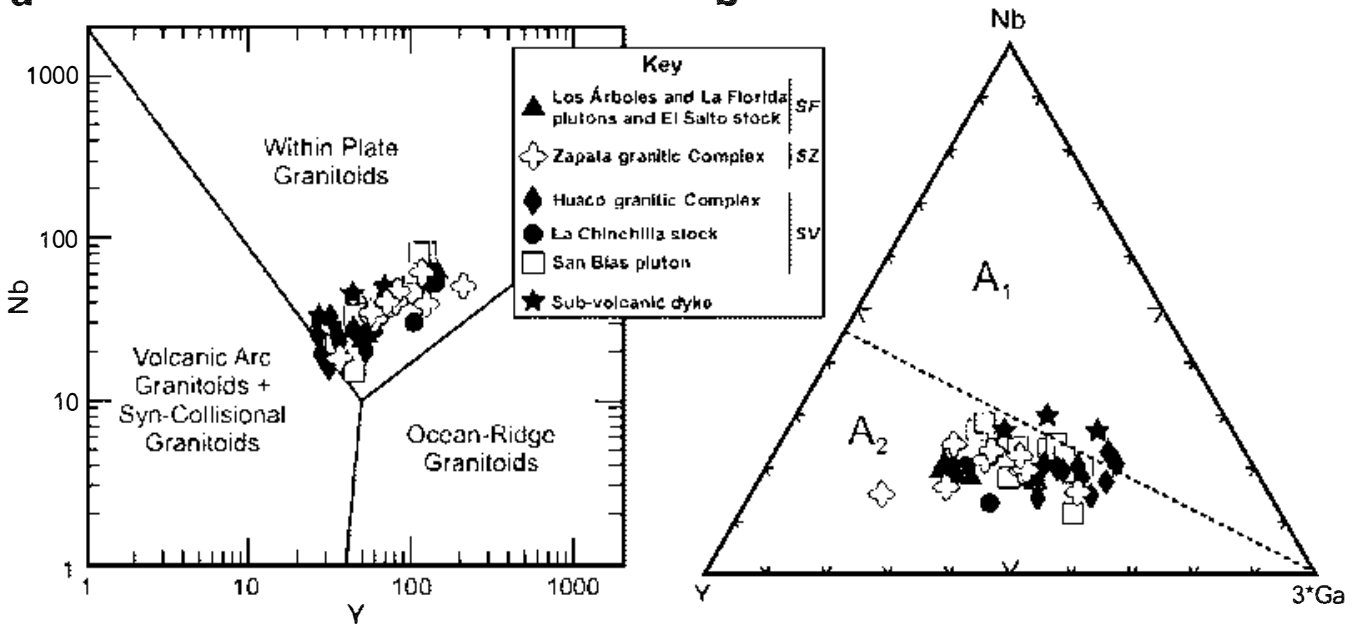

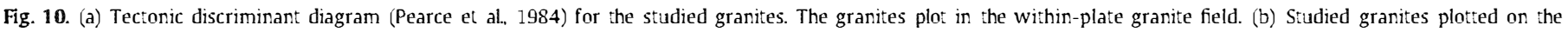

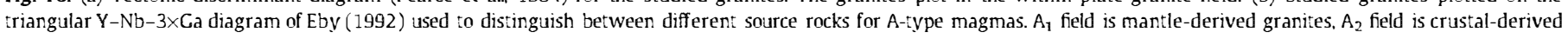
granites. 


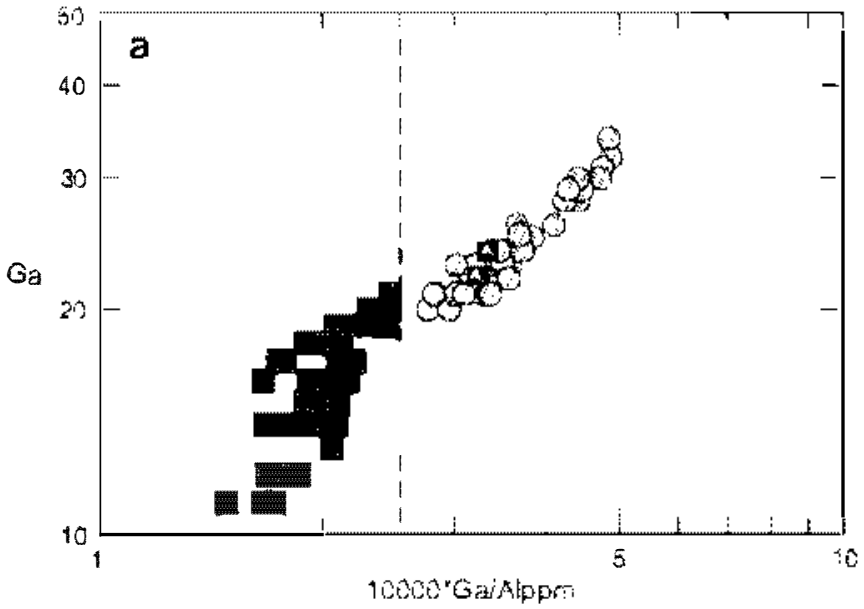

determined by older lineaments in the continents. The role of intracontinental shear zones and fault systems in the generation. ascent and emplacement of granitic magmas has also been etmohasized by Brown (1994) and Abdel Rahman (2006). The Early Carbonferous granitic plutons considered here are related to the promitent TIFA shear zone that underwent heaung coeval to Early Carboniferous magmatism. Although the history of this shear zone deserves further research, and normal and reverse sense of movement have been proposed (Grissom et al., 1998: Hockenreiner et al., 2003). we infer that the TTPA shear zone was active in the Early Carboniferous and could have controlled the emplacement of plutons. Melting of contirental crust was pubaby riggeted by mantle-derived magmas as inferred from isotope evidence given previously. Subsequent ascent of granitic magma was focused along the MPA shear zone. Heat advected to the upper crust by rising magmas favoured ductile deformation and recrystallization $(-342-328 \mathrm{Ma})$ within the shear zone. Cooing contmued long after pluton emplacement ( $-300 \mathrm{Ma})$.

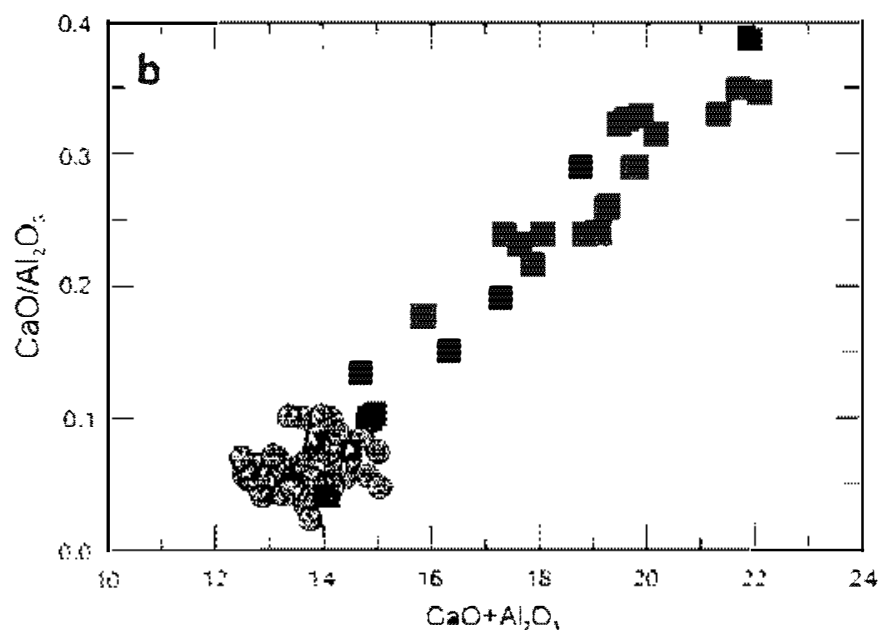

Fig. 12. $\mathrm{Al}_{2} \mathrm{O}_{3}, \mathrm{C}_{3} \mathrm{O}$, and $\mathrm{G}_{3}$ diagrams stiowing the compositions of the studied $\mathrm{A}-\mathrm{typ}$. granites (filled circle) and the Famacinian calc-alkaline granitoids (filled square). For the Famatinian calc-alkaline granitoids the $\mathrm{SiO}_{2}$ contens ranges from tonalites

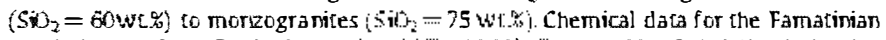

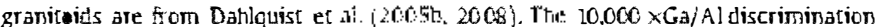
limit for A-type granites is from Whalen et al. (1987). $A_{i}$ and $A_{i i}$ are A-type average composition from konopelko etal (2007) and Chappell and Whice (1992), respectively.

suggested by Abdel Rahman (1994). Biotite minerals define three compositionally distinct fields in the $\mathrm{MgO}$ vs. FeO disirimitutory diagram of Abdel Rahman (1994). Biotites in the A-type sirnit there studied are iron-rich while biotites in calc-allcaline rocks ( 1 - and 5 . type gramles; are moderately enriched in $\mathrm{MgO}$ (lig. 9 ).

The calculated magma $\mathrm{F}$ and $\mathrm{Cl}$ content (toggether with the nog $*$ ) for these A-type granitoids (F-580-5600 ppm and $\mathrm{Cl}=90-$ $1180 \mathrm{ppm}$, see Section 5) is generally higher than that for calcalkaline granues ( $F-0-470 \mathrm{ppm}$ and $\mathrm{a}-0-250 \mathrm{ppm}$ ) although the values are not excessively high, ie, water is still a significunt part of the volatile component of the magmas. Thus, $\mathrm{F}$ and $\mathrm{a}$ content of igneous biotites seem to reflect the nature of their palcntal host magmas and are potential chemical elements to be used int discriminant diagrams.

\subsection{The the mai sourec for continentai lirhospherc anatexis}

As poinsed out by Brown (1994), the heat necessary for crustal anatexis may be the result of internal 1 relogrtuch heat poduction in over-thickened crust, muaplatmgiunderplating of mantle-derived magma, an enhanced flux from the mantle, or some combination of these mechanisms. Moreover, Black et al. (1984) showed that large faults play a major role during the emplacemenc of alkaline felsic magmas, as do transform faults whose poritinn may well have been

\section{Conclusions}

The whole-rock chemical and geochronological data allow us to determine unequivocally that these plutons are A-Iype granites empiaced during the Farly (arboniferous in the proto-Andean margin of Gondwana. The main conclusions of this study. which could be aplicable to other A-type grantes, are:

(1) A rype magmas represent variable mixtures of asthenospheric mantle and continental crust, and variations in the mixing ratios lead to different subtypes of A-type granite. In our study the magma source was primarily (and dominantly) mantle with subsequers crustal contamination.

(2) Shear zones (in our scudy the TIPA shear zone) pluy an important role, providing suitable conduits for ascending asthenospheric material and heat flux into the crust, a hypothesis that is in accord with other recent workabout A-type granires.

(3) Biotites have distinctive compositions with high $\mathrm{FeO} / \mathrm{MgO}$ ratios, $\mathrm{F}$, and $\mathrm{C}$. Thus, $\mathrm{FeO} / \mathrm{MgO}$ ratios together with $\mathrm{F}$ and $\mathrm{Cl}$ content of ignecuss biotites seem to reflect the nature of their patental host magmas and may be useful in identifying A-type gratutereds.

(4) Out petrogenetic model supports ptogressive fractional crystallization with dominant fractionation of feldspar and a source mineral assemblige enriched in plagioclase, explaining the distinctive negative Eu anomalies observed in A-type grantes.

\section{Acknowledgernewts}

Finantial suppotc was provided by the Yacimientos Minerales Riojatios (YAMIRI S.A , SECYT UNLaR grants Exp. No. 6977/07, and by

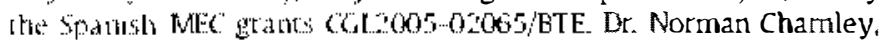

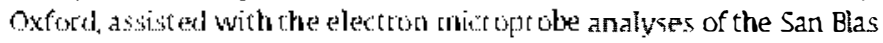
plut on biotites. The careful and thoingh reviews by two anonymous joutnal reviewers contributed significantly to the final form of the natulscript. We thank Andrew Kerr for his editorial handling of the mathutictipl

\section{Appendix A. Supptementary data}

Sw1phlemenary data associated with this article can be found, in the online version, at toi: $10.1016 / \mathrm{j}$ lithos 2009.11 .006

\section{References}

Abdel Rahman, A.M, 1994. Nature of biotiter from alkaline, calk-alkaline, and

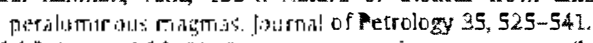

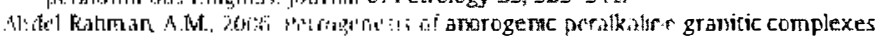

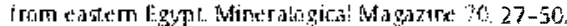


Aceñolaza, F.G, Miller, H. Toselli, A.J., 2000. Geología de la Sierra de Velasco, Provincia de La Rinja. Asger'ina. Cienwissersralliches Lateinamerika-Kolloquium. Profil, Band 18. Exterded A'sstratts in CD-ROM Scuttgart in 5pamish.

Alasmo, P.H, 2002. Caracterización Pererilógica del sector Nt. dr la Sierra de Velasca, la Rioja, Argenirra. Undergraduate thesis, "̈riversidad Nacional de Córdoba, Argentina (h) Sparish

Alasino, P F... xino Geología, petrología y geoquímica de los granitoides Famatinianos ubicados en el sector occidental, y su comparación con el sector central, a los $29^{\circ}$ de latioud sur del margen proto-andino de Gondwana, Sierras Pampeanas, Argentina. PhD. Universidad Nacional de Córdoba, Argentina (hı Spanish)

Alba, F, 2008. Caracterización perrolroguia y gennairica del plutón San Blas, provincia de La Rioja, Argentina. ̈̈rdergrad sale thes1s Universidad Nacional de San Juan, Argentina (In sporisk

Anderson, J.L, -983. Proterozoic anorogenic granite plutonism of North America Proterozoic Geology, Selected Prapers from an International Proterozoic symm sium: In: Mederis, LG., By'ers, C.W... Muckelsor, D.M, Shanks, W.C. (Eds.), Geological sncirly' of America ierrims, vol. 161, pe 139-152

Báez. M.A. Rossi de Toselli, J., Sardi, F., 2CO2. Consuderaciones preliminares sobre los granitoides del norte de la Sierra de Velasco, La Rioja, Argentina. In: Cabaleri, N., ringihlim. C, Linares, E, lopez de Luchi, M., Ostera, H., Panarello, H. (Eds.), Actas del XV Congreso Geologrco Argeniro, vol 2, pp. 69-74 (In Sparist:;

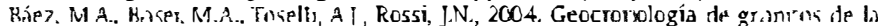
sierra de Velasco "Argen:ina;' rein:erprelacrón de la secuencia magmälica. Ac:as Simposio años ite geocronología en Mrasil, San Pablo, Brasil p $k$; in iparish)

Black R. Lancj,re. I, Bonin, B, 1984. The structural setting of alkalire complexes journal of Nfricir Earth scoences 3, 5-16.

Bor ir, B. 2607. A-iype granies and related rocks: evolution of a concept, problems and prospects $11.6051 \% 1-29$

Boynton, W.V, 1984. Geochemistry of the rare earth elements meteorites studies. In: Henderson, P. (Ed.), Rare tarth Fin ror $\mathrm{rl}$ Geochemistry. Elsevier. $57: 14 \mathrm{~ms}$

Brown, M, 1994. The generation, segregation, ascent and emplacement of grante niagma: the migmatite-to-crustally-derived granite connection in 1-skcred orogens. Earth-Science Reviews 36, 83-130.

Büttner, S.H. Glodny, J., Lucassen, F., Wemmer, K., Erdmann, S. Handler, R, Franz, G. 2005. Ordovician nuetarmphisn and plutonism in the Sierra de Quilmes mc:amorphic corıplcx.: implications for the tectonic setting of the northem Sierras Pampeanas 'W Aroprina' Lithos 83, 143-181.

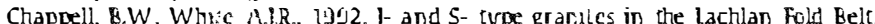
Transactions of the Rongin Society of Edinburgh: Farts Sciences 83, 1-26.

Dahlquist, J.A, Galindo, C.,2004 Geoqurrica isotópica de los granitoides de la sierra de Chepes: un modelo senlectónken y termal, implicancias pira el nrógern famatiniano. Revista de la Asociación Geolugica Argeruna 59, 57-69, in Sparist:).

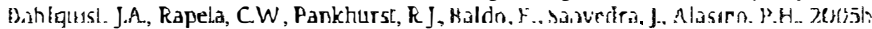
Losgramiondes de la sierra de Chepesy su comparacion cor: graricoides paleozortos

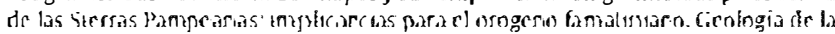
provircia de La Rroja - Precamionco-Paleuzoko infersur: in: Dahlquss:. I.A. Baldo

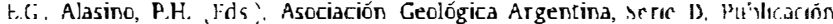
Especial, vol. 8, pp. 87-108 (In Spanish)

Dahlquist, J.A. Rapela, C.W, Baldo, E, 2005a. Petrogenesis ol cordhernle-tecarr.g S-type orar.teuts in Sierra de Chenes. Famatinian orogen, Argentina. Journal nf South Amencar. Ear:t. Scicreces 2C, 231-2j1

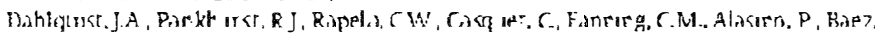

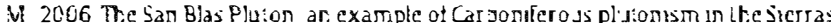

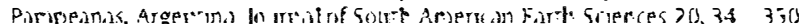

Dahlquist. J., Casquer, C., Alasino, P. Galirdo, C.. Grant, C., ivledrna C., 2007. A-iype

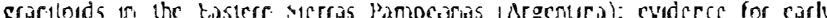
car'jonfferous a'zoried nfung? GEOS:R. An Ir:ernacional Congress or the Geology

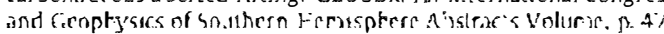

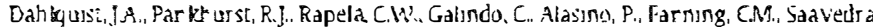

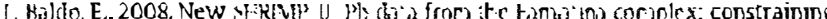
Early-Mid Ordovician Famatinian magmatism in the Sierras Pampeanas, Argentina. Gcologica Ac:a 6, 319-333.

De Finir, 11J Linn A.M, Schubert, G, 1991 . The continental crustal age distribution: me'hods of dc:ermining mantle separalior. age from Sm-Nd isotopic data and

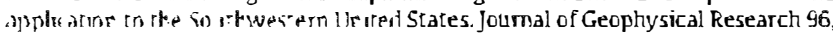
207:-2088.

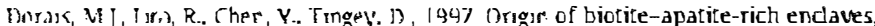

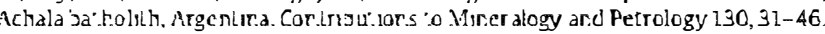

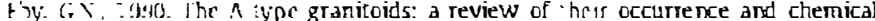
characerrsics and speculations on their petrogenesis. Lithos 26, 115-194.

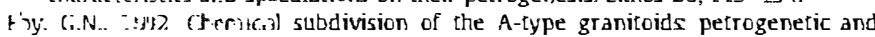
Eeciunk implications. Geology 20,641-644.

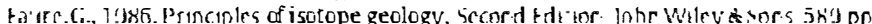

Frost, B.R. Barnes, C.G., Collins, WJ, Arculus, R.J. Ellis, D.I. Frost CD., 2001. A geochemical classification for granitic rocks. Journal of Perrology 42, 2033-2048.

Grissom, G.C., Debari, S.M, Lawrence, WS, 1998. Geology of the Sierra de Fiambala, northwestern vigen:rra implicalions for early Palaezoic Andean tectonics. In: Pankhurst, R.J, Ranpeir. C.W' (Eds.), The Proto-Andean Mirgin of Gondwana

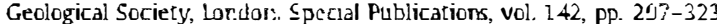

Grosse, P, Sällner, F, Baéz, M.A., Tospll. A.J., Rossi.J.N., de la Rrusi. J.D. 2009. Lower Carboniferous posl-oroger..1 grar.ules in central-eastem Sierra de Velasco, Sierras Pampeanas, Arapnriri. : Pls monazite geochronology and Sr-Nd isotopes. Inierracional gournal of Earth Science 98, 1001-1025.

Höckenteiner, M, snllecr. F., Miller, H., 2003. Da:org the TIPA shear zone: an early Devonian terrane boundary between the Famairian and Pampean systems (NW Argentina). Joumal of South America Earth Sciences 16، 4.566. cenhower, J., London, D, 3997. Partusunung of fluorine and chlorine between biotite

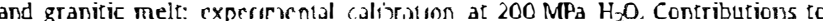
Mineralogy and Pe:rulog.' 127, 17-25.

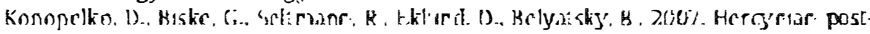
collisional A-Cype granties of ite kokstaal Range. Southerr Tser St ar, Kyrgyzstar Lithos $97,140-160$

Kretz, R., 1983 . Symbols for rock-forming minerals. American Mineralogist 68 $277-279$.

Lambías, F J Sato, A.ML, Ortiz Suárez, A., Prozzi, C, 1998. The granitoids of the Sierra de San Luls. The Proto-Andean margin of Gondwana: hy: Pankhurst, R.J, Rapela, CW. (Eds.), Geological Society of London, Special Publication, vol. 142, pp. 325-341.

Loiselle, MC. Wones, D.R., 1979. Characteristics and origin of aroroger.k granites. Abstracts of pupers to be presented at the Annual Meetings of the Cieningsial Society of Amerita and Associated Societies, San Diego, Calfifornia, November $3-8$, vol. 11, p. 468.

lópez de luchi, MG., Siegesmund, 5., Wemmer, K.. Steenker. A. Yaumarr. R., 2607.

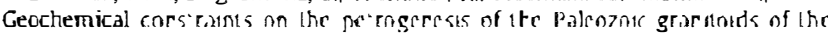
Sierra de San luis, Sierras Pampeanas, Argentura. Journal of Soluit Anerkicar: Earit science $24,138-166$

Malvin. D.J, Drake, MJ, 1987. Experomental determination nf irysinfiriel parrurmonng of $\mathrm{Ga}$ and $\mathrm{Ge}$ in the system forslerile-arortbale-dhopside Geochenica el Cosmochimica Acta 51, 2177-2128.

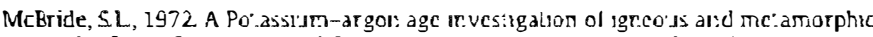

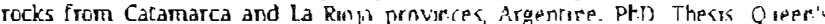
"niversty 'unpublished' Ontario. Canada

Mckride, SI.. Carlks, J6.. (Jark. A.H., Farrar, E, 1976. Paleozoic radiometric age provir ces in the Andean basement, latitudes $25^{\circ}-30^{\circ} \mathrm{S}$. Earth and Planetary Science |rines: 29, 373-383

Miller, H., Sölner, F., 2005. The Famatina complex? 'WW-Argenar al: 'jack-docking uf an

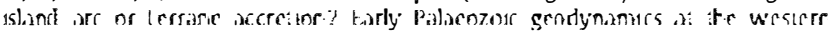

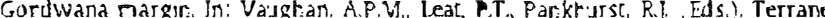
processes at the margins of Gondwana. Geological Society of I.nndinn. Special Publication, val. 246, pp. 24l-256.

Miller, CF. McDowell, S.M. Mapes, R.W, 2003. Hot and cold grarsics? Implscatons of zircon saturation temperatures and preservation of irharramis Genisga 31 , 529-532.

Miyashiro, A., 1978. Nature of alcalic volcanic rock series. Contributions to Mineralogy and Petrology 66, 91-14.

Muñoz, JL., 1984. F-OH and $\mathrm{Cl}-\mathrm{OH}$ extharge in nuras winh anpluarmo on hydro:termal ore deposts. in: Baley, S.W. (Ed.) Review's in Thresalugh: Mocas,

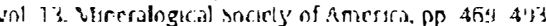

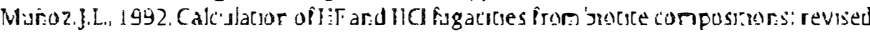

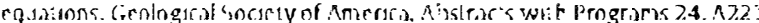

Nagasawa, Y". Schreezler, C.C. 1971. Partutsoning of rare Eartt: alkalt, and alkainne Earth

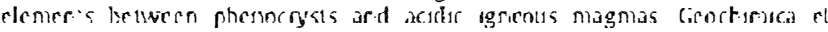
Cosmoctimica Acta 35. 953-968.

Nakamura, N., 1974. Determination of REE, $\mathrm{Ba}, \mathrm{Mg}, \mathrm{Na}$ and $\mathrm{K}$ in carbonaceous and ordinary chondrites. Geochimica et Cosmochmica Acta 38, 757-773.

Pankhurst, R.J, Rapela, C.W, Sazwedra. J. Baldo, E.G., Dahlquisc, J.A., Pascua, l., Far:nrng CM, 1998. The Famatinian arc in to central Sierras Pampeanas: an early to $r$ w Ordovician continental arc on the Gondwana margrr. The Proto-Andean Margin of Gondwana: In: Pankhurst, R., Rapela, CW. Fits Geologkal Society, London, Special Publications, vol. 142, pp. 343-367.

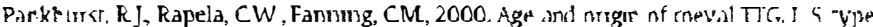
grarstes in the Famatinian belt of NW Argentria. Transacons: of the Royal Socte:y

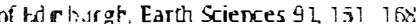

Patiño Douce, A.E.. 1997. Generation of me:alumir:ous 4-iype grarices by low-pressure melting of calc-alkaline grarilnids cicoing: 2.5. 14 is $7 / 46$.

Patiño Douce, A.E., 1998. What do experiments :ell us a'so to the relanve cor:mburor:s

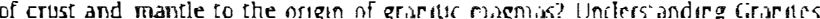
Integrating New and Classical Tecrnayises: In: Casiro. A. Ferráardez, C., Vigneresse, JL. Eds i Gcological Socic:y ot Lor:don Special P.jblica:ior.s. vol :68. pp 5j-75

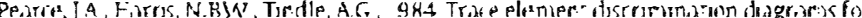

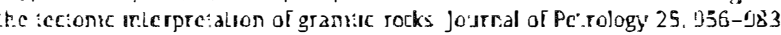

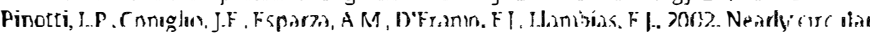
plutons craplaced by s:oping al shallow cristal lcucls. Cerro Aspcro batholith

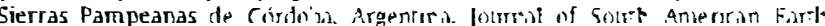
Sciences 15, 25 1-265.

Pinotti, L, Tubía, J.M. D'Eramo, F., Vegas, N. Sato, A.M, Coniglio, J. Nrar guren. A. 2006 Structural iresplay betweer pluions during the construction of a batholith (Cerro

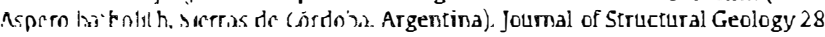
$834-8+9$.

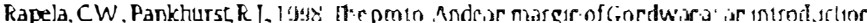
The proto-Andean marger: of Gondwana: In: Par ks'usst. RJ.. Rapela, CW: (Eds:

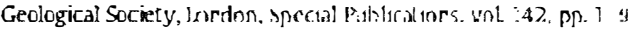

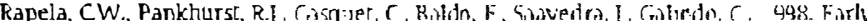
evolution of the proloss:dear. margin of Sojt: America Gcology 26. 707-7:0.

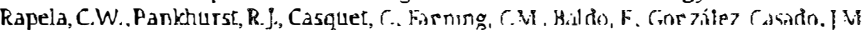
Galindo, C, Dahlquist, A. 2007. The Río de la Plata craton and the assembly of SW Gondwana. Earth-Science Reviews 83, 49-82.

Rapela, C.W., Baldo, E.G., Pankhurst, R.J., Fanning, CM, 2008. The Devonian Achala batholith in the Sierras Pampeanas F-rich aluminous A-type granites. V South American Sympostian on lsotope Geology, Proceedings in CD-ROM, Paper 53. San Carlos de Rirtloche. Argerlint

Rapela, CW, Pankhurst, R.j. Casquet, C, Baldo, E, Galindo, C, Fanning, C.ML, Dahlquist, 1.A. ir press. Circnuille agc magmatism of the Western Sierras Pampeanas, 
Soutrerr: South smenca: LI-Pb SI RIMP datirg ard tectonic affinities: Journal of

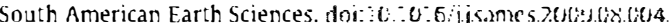

Rollinson, H.R., 1994. “jir:g geochemutal daid evaliation, presentatuon, interpretation. Longman Scientific \& Ifechrical Editions, singapore. $452 \mathrm{pm}$.

Saavedra. J., Baldo, E... Pankhurst, R. J., Rapela, C.W., Murra. J. _998. El granito Capilla de Monte (Sierras Pampeanas de Córdoba. Argentina): edad, geoquirnica. ginesis y especialización metalogénica. 10 Congreso Latinoamericano tie (imollogía. As:ai vol. 2, p. 372 (In Spanish).

Sihnepers. R., 2000. Granites of the Saldania mobile belt, South Africa: radioelements and $P$ as discriminators applied to metallogeny. Journal of Geochemical Exploration 68, 69-86.

Shand, S.]., 192\%. The eruptive rocks. John Wiley, New York. 36C pp

Siegesmund, S., Steenken, A., Litph? de Luchi, M.G., Wemmer, K., Foffenane. A. Mosst S., 2004. The Las Chacras-Potrerilless batholith (Pampean Ranges, Argentral

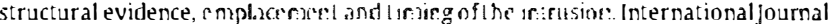
Earth Sciences 93, 23-43.

sim., J.P. Ireland, T.R., Camacho, A., Lyons, P., Pieters, P.E., Skirrow, R.G, S tuart-Smith, P.G. 1998. U-Pb, Th- $\mathrm{Pb}$ and $\mathrm{Ar}-\mathrm{Ar}$ geochronolugy from the southern Sierras Pampeanas irger:ina: irmplications for :pe Palacozoic tectonic evolution of the western Grindwiand rourgin. Ir: Pankhuss: R.J. Rapela, C.W. (Eds.), The Proto-Andean Margir ol Gondwar.a. Gcological socicl; oflordon. Special Publication. vol. 142, pp. 259-281.

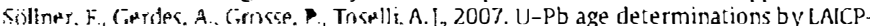
Vis on zircors of the lluaro grarile. Sierra de Velasco (NW-Argentina): a long term history of melt arenry with an igneous body. Proc XX Colloquium Latin America Earth Sciences, vol. 1, pp. 57-58. Kiel. Gcrman;

s:ciger. R.H., Jäger, E., 1977. Subcommission of genchronnolngy: corventinn or :tes use of decay' constants in geo and cosmochronology. Eastr: ard Planeiary Scuence Leiters $36.35^{\prime} ! 362$
Streckeisen, A, 1976. To each plutonic rock its proper name. Earth Science Reviews 12 , $1-33$.

Stuart Smith, P.G., Camacho, A, Sims, J.P., S kirrow, R.G., Pieters, P.E., Black, L.P., Miró, R, 1999. U-Pb. Th- $\mathrm{Pb}$ and $\mathrm{Ar}-\mathrm{Ar}$ geochronology from the southern, 1999. Uraniuml.ead dating of felsic magmatic cycles in the southern Sierras Pampeanas, Argentina: implications for the tectonic development of the protn Andean Gondwana margin. Laurentia Gondwana connections before Pangea: In: Ramos, V.A. Keppe, I.D. (Eds.). Gcological Sorizey of America. Special Paper, vol. 336, pp. 87-114.

S.r. S.S. Mithnnoigh. W.F. 1989. Chemical and isotopic systematics of oceanic basalts; implirzitions for mantle composition and processes. Magnatism in the ocean '2.xins: In: Saunders, AD., Norry, M.J. (Ed s.), Geological Society of London, vol. 42, pp. 313-345.

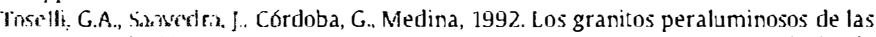
sierras de Vinquis. Cerro Negro, y Zapata (Sierras Pampeanas), provincia de

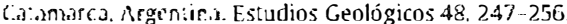

Watsor. E. I:arrison. T.M., 1983. Zircon saturation revisited: temperature and composition effects in a variety of crustal magma types. Earth and Planetary Science Letters 64, 295-304

Whalen, J.B., Currie, K.L., Chappell, B.W., 1987. A rype granites: geochemicil characteristics, discrimination and petrogenesis. Contributions to Mineralogy and Petrology 95, 407-419.

Wilson, M., 1989. Igneous Petrogenesis: A GlobalTectonic Approach. Chapman \& Hall, London. $446 \mathrm{pp}$. 